\title{
On the Ginzburg-Landau model of a superconducting ball in a uniform field
}

\section{Sur le modèle de Ginzburg-Landau pour une boule supraconductive dans un champ uniforme}

\author{
Stan Alama ${ }^{1}$, Lia Bronsard ${ }^{*, 2}$, J. Alberto Montero $^{3}$ \\ Dept. of Math. \& Stats., McMaster University, Hamilton, ON, Canada L8S 4 K1
}

Received 24 August 2004; received in revised form 28 February 2005; accepted 17 March 2005

Available online 7 December 2005

\begin{abstract}
We consider the three-dimensional Ginzburg-Landau model for a solid spherical superconductor in a uniform magnetic field, in the limit as the Ginzburg-Landau parameter $\kappa=1 / \varepsilon \rightarrow \infty$. By studying a limiting functional we identify a candidate for the lower critical field $H_{c_{1}}$, the value of the applied field strength at which minimizers first exhibit vortices. For applied fields of this strength we show the existence of locally minimizing solutions with vortices located along a diameter of the sphere parallel to the applied field direction. To analyze these problems we use a combination of techniques, involving least perimeter problems, weak Jacobians and rectifiable currents, and special Hodge decompositions.

C) 2006 L'Association Publications de l'Institut Henri Poincaré. Published by Elsevier B.V. All rights reserved
\end{abstract}

\section{Résumé}

Nous étudions la limite quand le paramètre de Ginzburg-Landau $\kappa=1 / \varepsilon \rightarrow \infty$ pour le modèle de Ginzburg-Landau en trois dimension dans le cas d'une boule placée dans un champ magnétique uniforme. Nous identifions une fonctionnelle limite qui nous permet de trouver le premier champ critique $H_{c_{1}}$, c'est à dire le champ au dessus duquel les minimiseurs commencent à presenter des vortex. Nous montrons qu'il existe des solutions localement minimisantes ayant des vortex le long du diamètre de la boule qui est parallèle au champ appliqué quand sa norme est de l'ordre de $H_{c_{1}}$. Nous nous servons de techniques provenant de la théorie de la mesure géométrique, incluant les jacobiens faibles et les courants rectifiables, ainsi que de techniques provenant de problèmes de minimisation de périmètre.

(C) 2006 L'Association Publications de l'Institut Henri Poincaré. Published by Elsevier B.V. All rights reserved

MSC: 35J50; 35B25

\footnotetext{
* Corresponding author.

E-mail addresses: alama@mcmaster.ca (S. Alama), bronsard@mcmaster.ca (L. Bronsard), alberto@math.toronto.edu, monteroj@math.mcmaster.ca (J.A. Montero).

1 Supported by an NSERC Discovery Grant.

2 Supported by an NSERC Discovery Grant.

3 Current address: Department of Mathematics, University of Toronto, Toronto, ON, Canada M5S 3G3
} 


\section{Introduction}

In 1933 Meissner and Ochsenfeld performed an experiment which exposed a solid spherical superconductor to an external magnetic field, and described the well-known Meissner effect whereby the superconductor expels the field (and levitates in its presence). Some years later, Abrikosov studied the behavior of the type-II superconductors and predicted the nucleation of vortices (where superconductivity is lost) in sufficiently strong external fields. (See [25].) In this paper we revisit this setting in the mathematical context of the Ginzburg-Landau model. We consider a spherical superconductor in a uniform external field, and study vortices which appear near the lower critical field, the smallest value of the external field strength at which minimizers exhibit vortices, in the extreme type-II limit as the GinzburgLandau parameter $\kappa \rightarrow \infty$.

We start with the Ginzburg-Landau model. Let $\Omega=B_{R}(0)$ the solid spherical ball of radius $R$ centered at the origin. The external magnetic field $h_{\text {ap }}=H \hat{e}_{3}$ is assumed to be of constant strength $H$ and directed (without loss of generality) in the direction of the $x_{3}$-axis. Superconductivity is described by a complex valued order parameter $u \in H^{1}(\Omega ; \mathbb{C})$. The square modulus $|u|^{2}$ measures the density of superconducting electrons. The magnetic field is determined by the external field and by the supercurrents and its interaction with the superconductor is mediated by the vector potential, $A: \mathbb{R}^{3} \rightarrow \mathbb{R}^{3}$, so that $h=\nabla \times A$ is the local field at any point in $\mathbb{R}^{3}$. The Ginzburg-Landau model then takes the form of an energy that a superconducting configuration must minimize (at least locally) in order to be stable. This energy is given by

$$
G_{\varepsilon}(u, A)=\int_{\Omega}\left\{\frac{1}{2}|(\nabla-\mathrm{i} A) u|^{2}+\frac{1}{4 \varepsilon^{2}}\left(1-|u|^{2}\right)^{2}\right\} \mathrm{d} x+\int_{\mathbb{R}^{3}}\left|\nabla \times A-h_{\mathrm{ap}}\right|^{2} \mathrm{~d} x .
$$

The parameter $\varepsilon>0$ is related to the Ginzburg-Landau parameter $\kappa$ by $\varepsilon=1 / \kappa$, and for strongly type-II superconductors (such as most high- $T_{C}$ materials) $\varepsilon$ will be very small.

Some care must be taken to define an appropriate space for $(u, A)$, since the functional is gauge invariant $\left(G_{\varepsilon}(u, A)=G_{\varepsilon}\left(\mathrm{e}^{\mathrm{i} \phi} u, A+\nabla \phi\right)\right.$ for any $\phi$ sufficiently smooth and integrable) and we require a space in which the energy will be coercive in the norm. (See [9].) This choice will be made precise in Section 5.

In this paper we look for stable critical points of $G_{\varepsilon}$ that develop line vortices in the singular limit $\varepsilon \rightarrow 0$. Since vortices are regions where the material is no longer superconducting, it is natural to think them as the regions where $|u|^{2}=0$. A vortex solution for us will be a critical point $\left(u_{\varepsilon}, A_{\varepsilon}\right)$ of $G_{\varepsilon}$ for which $u_{\varepsilon}$ vanishes somewhere in $\Omega$. Physically, one expects that for an applied field $h_{\mathrm{ap}}^{\varepsilon}=\mathrm{O}(|\ln \varepsilon|)$, the Meissner effect should cease and vortices should begin to appear in the domain. With a constant applied field along the $\hat{e}_{3}$-direction, a natural candidate for the line vortex is the vertical diameter of the ball. We will confirm this physical principle in that we will show that for $h_{\mathrm{ap}}^{\varepsilon}=$ $\lambda|\ln \varepsilon| \hat{e}_{3}$ and $\lambda>0$ large enough, there exist stable vortex solutions (indeed local minimizers) to $G_{\varepsilon}(u, A)$ for all $\varepsilon>0$ small enough; these solutions will have vortices converging in a weak sense to the vertical diameter as $\varepsilon \rightarrow 0$. Moreover, since our superconductor is spherical we can find an explicit estimate for how big $\lambda>0$ should be. This raises a natural conjecture in the form of an explicit asymptotic form for the critical field $H_{c_{1}}$ at which the transition from the Meissner phase to the mixed phase occurs.

In order to motivate and explain more precisely our results, we present a formal derivation of the limiting energy based on the useful identity introduced by Bethuel and Rivière [5],

$$
G_{\varepsilon}(u, A)=E_{\varepsilon}(u)-\int_{\Omega} A \cdot j(u) \mathrm{d} x+F_{h_{\mathrm{ap}}^{\varepsilon}}(A)+\int_{\Omega}\left(|u|^{2}-1\right)|A|^{2} \mathrm{~d} x,
$$

with

$$
\begin{aligned}
& j(u):=\operatorname{Im}\{\bar{u} \nabla u\}, \\
& E_{\varepsilon}(u):=\frac{1}{2} \int_{\Omega}\left\{|\nabla u|^{2}+\frac{1}{2 \varepsilon^{2}}\left(|u|^{2}-1\right)^{2}\right\},
\end{aligned}
$$




$$
F_{h_{\mathrm{ap}}^{\varepsilon}}(A):=\frac{1}{2}\left[\int_{\Omega}|A|^{2} \mathrm{~d} x+\int_{\Omega}\left|\nabla \times A-h_{\mathrm{ap}}^{\varepsilon}\right|^{2} \mathrm{~d} x\right] .
$$

In the regimes we consider the applied field is weak, $\left|h_{\mathrm{ap}}^{\varepsilon}\right| \ll 1 / \varepsilon^{2}$, and it will follow that the last term in (1.2) will be very small and can be neglected.

The first term $E_{\varepsilon}$ in (1.2) measures the energy of the vortices, and has been extensively studied for general smooth domains $\Omega \subset \mathbf{R}^{n}$, for all dimensions $n \geqslant 2$. Much previous work has concentrated on a Dirichlet problem (see for instance [3,4,17,20,21]), in which the presence of vortices in $\Omega$ is assured by imposing a singular Dirichlet boundary condition. In these papers it is shown that as $\varepsilon \rightarrow 0$ singularities form which are objects of codimension-2, and these vortices tend to minimize the total length functional. This connection to the total length functional was cemented by the subsequent work of [14] and [2] in the context of Gamma-convergence. For $\Omega \subset \mathbf{R}^{3}$ the results of these two papers yield that

$$
\frac{E_{\varepsilon}}{|\ln \varepsilon|} \rightarrow \pi L
$$

in the $\Gamma$-sense, where $L$ denotes the total length of the singularities (defined appropriately in terms of rectifiable currents.)

Both [14] and [2] show that in the study of the $\Gamma$-limit of $E_{\varepsilon}$ the correct tool to identify the limiting vortices is not the "momentum" $j(u)$ but its distributional curl, the Weak Jacobian of $u$,

$$
J u:=\frac{1}{2} \nabla \times j(u) .
$$

Indeed, for $\Omega \subset \mathbf{R}^{3}$ an important result of Jerrard and Soner [14] implies that when $\left(u_{\varepsilon}\right)$ is a family of functions with $E_{\varepsilon}\left(u_{\varepsilon}\right)$ bounded by $|\ln \varepsilon|$ then the Jacobians $J u_{\varepsilon}$ converge in a weak sense to an integer multiplicity rectifiable 1-current.

Returning to the decomposition (1.2) and our derivation of a limiting energy, if we hope to find solutions $u_{\varepsilon}$ with individual vortices, the above discussion suggest that we consider a regime where $E_{\varepsilon}\left(u_{\varepsilon}\right)=\mathrm{O}(|\ln \varepsilon|)$. Then by the result of [14] the associated Jacobians $J\left(u_{\varepsilon}\right)$ will converge to a rectifiable limit. In this situation the interaction term $\int_{\Omega} A \cdot j(u)$ should also be of order $\mathrm{O}(|\ln \varepsilon|)$, to balance the cost of vortices from $E_{\varepsilon}$. This suggests that the appropriate applied field $\left|h_{\mathrm{ap}}^{\varepsilon}\right|=\mathrm{O}(|\ln \varepsilon|)$, since the form of $F_{h_{\mathrm{ap}}^{\varepsilon}}$ suggests that the magnetic field $h_{\varepsilon}=\nabla \times A_{\varepsilon}$ will be of the same order of magnitude as $\left|h_{\mathrm{ap}}\right|$. Note that in this case we expect $F_{h_{\mathrm{ap}}^{\varepsilon}}\left(A^{\varepsilon}\right)=\mathrm{O}\left(|\ln \varepsilon|^{2}\right)$ is of higher order in the expansion. Minimizing this term independently (see Theorem 3.1) gives a solution $A_{0}$ to London's equation, which approximates the actual minimizer $A_{\varepsilon}$ to highest order. We then eliminate this term and consider letting $\varepsilon \rightarrow 0$ in the expression

$$
\frac{1}{|\ln \varepsilon|}\left(G_{\varepsilon}\left(u_{\varepsilon}, A_{\varepsilon}\right)-F_{h_{\mathrm{ap}}^{\varepsilon}}\left(A_{0}\right)\right) \simeq \frac{1}{|\ln \varepsilon|}\left(E_{\varepsilon}\left(u_{\varepsilon}\right)-\int_{\Omega} A_{0} \cdot j\left(u_{\varepsilon}\right) \mathrm{d} x\right) .
$$

To pass to the limit in (1.5) we need to rewrite the interaction term in terms of the Jacobian of $u_{\varepsilon}$. We decompose $A_{0}=\nabla \times B_{0}+\nabla \phi_{0}$ for a suitable vector field $B_{0}$ with $B_{0} \times \nu=0$ on $\partial \Omega$, and scalar function $\phi_{0}$. The existence and properties of various versions of this decomposition have been studied at least since 1940 (see for instance [26,16] and [11]). In Section 2 we recall a specific version of it that best suits our purposes, taken from [4]. Borrowing a nifty trick from [13] we eliminate the $\nabla \phi_{0}$, and essentially integrate by parts to obtain an equivalent form in terms of the Jacobian,

$$
\int_{\Omega} A_{0} \cdot j\left(u_{\varepsilon}\right) \mathrm{d} x=-2 \int_{\Omega} B_{0} \cdot J\left(u_{\varepsilon}\right) \mathrm{d} x .
$$

Assuming for instance that we have a family $\left\{\left(u_{\varepsilon}, A_{\varepsilon}\right)\right\}$ with $E_{\varepsilon}\left(u_{\varepsilon}\right) \leqslant C|\ln \varepsilon|$, by [14] the Jacobians converge $J\left(u_{\varepsilon}\right) \rightarrow \pi T$, with $T$ an integer multiplicity rectifiable 1-current, and hence both terms on the right-hand side of (1.5) converge. Assuming the applied field is of the form $h_{\mathrm{ap}}=\lambda|\ln \varepsilon| \hat{e}_{3}$, this formal procedure suggests a candidate for a limiting energy of vortex lines:

$$
\mathcal{G}_{\lambda}(T)=\pi M(T)-2 \pi \lambda T\left(B^{*}\right),
$$


where $B^{*}=B_{0} /\left|h_{\text {ap }}\right|, M(T)$ is the mass of the current $T$ (roughly speaking, the total length of the vortex curves) and $T(\cdot)$ gives the action of the 1 -current $T$ on vector fields in $\Omega$.

We study the line energy in two different ways in Section 4 . In both of them the condition $\Omega=B_{R}(0)$ will be crucial, since it will provide means to explicitly find the field $B^{*}$ present in the energy. For our first analysis we choose a setting which allows us to consider global minimizers of $\mathcal{G}_{\lambda}$ in a particular class of curves. To describe briefly how this is done, note that the symmetry of the sphere ensures that planar curves will have lower energy than space curves. This allows us to think of the vortex curve optimally partitioning a two-dimensional cross-section

$$
B_{R}^{2}:=\left\{x=\left(x_{1}, x_{2}, x_{3}\right): x_{1}^{2}+x_{3}^{2}<R^{2}, x_{2}=0\right\}
$$

of $\Omega$. This partitioning problem may then be set in $\operatorname{BV}\left(B_{R}^{2} ;\{0,1\}\right)$, as the vortex defines the boundary of a set $E$ of finite perimeter in $B_{R}^{2}$. A similar reduction has been used to study vortices in Bose-Einstein condensates in [1].

We show that the global minimizer in this BV sense undergoes a transition at a critical value of the parameter $\lambda$,

$$
\lambda^{*}:=\frac{\sinh R}{3 \int_{0}^{R}(\cosh r-(\sinh r) / r) \mathrm{d} r} .
$$

When $\lambda<\lambda^{*}$ the global minimizer of the line energy $\mathcal{G}_{\lambda}$ is the vortexless Meissner state, while when $\lambda>\lambda^{*}$ the minimizer has a single vortex along the vertical diameter of the ball $\Omega$. Proposition 4.2 gives a precise formulation of this result. Thus we predict that the leading term in the expansion of the lower critical field $H_{c_{1}}$ in the sphere is

$$
H_{c_{1}} \sim \lambda^{*}|\ln \varepsilon| .
$$

We note that in two dimensions a much more detailed description of the lower critical field and the number and locations of vortices has been obtained in a series of papers by Sandier and Serfaty (see [24,23].)

Unfortunately, the formal limiting procedure outlined above does not allow us to conclude that the Jacobians $J\left(u_{\varepsilon}\right)$ of a family $\left\{\left(u_{\varepsilon}, A_{\varepsilon}\right)\right\}$ of global minimizers of $G_{\varepsilon}$ converge to an integer multiplicity rectifiable 1-current, as we lack the control of $\left(E_{\varepsilon}\left(u_{\varepsilon}\right)\right) /|\ln \varepsilon|$ required by [14]. Nevertheless, in Section 6 we present a partial result concerning the transition of global minimizers from the Meissner to the mixed state. In Theorem 6.1 we show the existence of an explicit value $\lambda_{m}^{*}<\lambda^{*}$ so that when $\lambda<\lambda_{m}^{*}$ the global minimizers of $G_{\varepsilon}$ have no vortices as $\varepsilon \rightarrow 0$. Thus $\lambda_{m}^{*}|\ln \varepsilon|$ defines a lower bound for the critical field, $H_{c_{1}}$. As functions of the radius of the ball $R$, these values accord for very large radii: as $R \rightarrow \infty, \lambda_{m}^{*} / \lambda^{*} \rightarrow 1$ and both $\lambda_{m}, \lambda^{*} \rightarrow \frac{1}{3}$. For this result, we use the weak Jacobians method of [14] and we extend a compactness result for Jacobians due to Sandier and Serfaty [22] (see Theorem 6.4).

To complement these results we construct stable solutions with vortices as local minimizers of $G_{\varepsilon}$ for $\lambda>\lambda^{*}$, using the methods of Montero, Sternberg, and Ziemer [19] and of Jerrard, Montero, and Sternberg [13] based on the $\Gamma$-convergence scheme of Kohn and Sternberg [15]. For this procedure we first show that for $\lambda_{\text {ap }}>\lambda^{*}$ the diameter (taken with any arbitrary multiplicity $m$ ) is an isolated local minimizer of the line energy $\mathcal{G}_{\lambda}$ in a suitable topology on the space of integer multiplicity rectifiable 1-currents. This will also depend on the explicit expression available for $B^{*}$ in the case $\Omega=B_{R}(0)$ and comprises the second part of Section 4. In Section 5 we show that there are indeed local minimizers of $G_{\varepsilon}$ with Jacobians converging to the vertical diameter with appropriate multiplicity. We recall that, given an isolated local minimizer of the limit energy $\mathcal{G}_{\lambda}$, the Kohn-Sternberg approach produces local minimizers to $G_{\varepsilon}$ (and $\varepsilon>0$ small enough) for any smooth simply connected $\Omega \subset \mathbf{R}^{3}$. The problem in a general domain would be first find the field $B^{*}$ involved in the expression we have for the limiting energy, and then seek a candidate for an isolated local minimizer of this energy.

To describe our result precisely we need to introduce some notation. Let $S_{1}$ denote the vertical diameter of the ball $\Omega$ thought of as a 1-current, that is $S_{1}$ acting on a vector field $B$ in $\Omega$ via

$$
S_{1}(B)=\int_{-R}^{R} B(0,0, z) \cdot \hat{e}_{3} \mathrm{~d} z,
$$

and set

$$
S_{n}=n S_{1},
$$


the same diameter but with multiplicity $n \in \mathbb{Z}$. We denote by $W_{T}^{1, p}\left(\Omega ; \mathbb{R}^{3}\right)$ the Sobolev space of vector fields $B$ which satisfy the boundary condition $B \times v=0$ on $\partial \Omega$. We then define a family of open neighborhood of $S_{n}$,

$$
\mathcal{O}_{\delta}:=\left\{(u, A) \in \mathcal{W}:\left\|S_{n}-\frac{1}{\pi} J(u)\right\|_{1,4}^{*}<\delta\right\}
$$

where $\|\cdot\|_{1,4}^{*}$ is the norm in $\left(W_{T}^{1,4}\left(\Omega ; \mathbb{R}^{3}\right)\right)^{*}$, and $\delta>0$. We prove:

Theorem 1.1. Let $h_{\mathrm{ap}}^{\varepsilon}=\lambda|\ln \varepsilon| \hat{e}_{3}$ with $\lambda>\lambda^{*}$. Then for every sufficiently small $\delta_{0}>0$ there exists $\varepsilon_{0}>0$ and a family of local minimizers $\left(u_{\varepsilon}, A_{\varepsilon}\right)_{0<\varepsilon<\varepsilon_{0}}$ of $G_{\varepsilon}$ in $\mathcal{O}_{\delta_{0}}$. The distributional Jacobians $J\left(u_{\varepsilon}\right)$ associated to these minimizers satisfy $\frac{1}{\pi} J\left(u_{\varepsilon}\right) \rightarrow S_{n}$ in $\left(W_{T}^{1,4}\left(\Omega ; \mathbb{R}^{3}\right)\right)^{*}$. Moreover, for any $\eta>0$ there is $\varepsilon_{0}>0$ such that, for any $0<\varepsilon \leqslant \varepsilon_{0}$ one has

$$
\operatorname{supp}\left(S_{1}\right) \subset\left\{x \in \Omega: \operatorname{dist}\left(x, N_{1 / 2}^{\varepsilon}\right) \leqslant \eta\right\},
$$

where

$$
N_{1 / 2}^{\varepsilon}=\left\{x \in \Omega:\left|u_{\varepsilon}(x)\right| \leqslant 1 / 2\right\} .
$$

In other words, we find solutions of the Ginzburg-Landau system with vortices which are close (in the given sense of currents) to the diameter. Since multiple degree vortices are considered to be unstable, we expect that the multiplicity $n \geqslant 2$ solutions will have $n$ distinct vortex lines, but these will approach the diameter of the ball in the $\varepsilon \rightarrow 0$ limit. In the two-dimensional setting Serfaty [24] has shown that this is indeed the case, with a distance between the different vortices on the order of $|\ln \varepsilon|^{-1 / 2}$.

\section{Some facts about vector fields}

We introduce in this section the main Sobolev space of vector fields we use in this paper. We also record some facts about Hodge decomposition for vector fields in $\mathbb{R}^{3}$ for future reference. In this section $\Omega \subset \mathbb{R}^{3}$ can be any bounded, smooth simply-connected domain. We first recall the following lemma from [16].

Lemma 2.1. For $A \in C_{0}^{\infty}\left(\mathbb{R}^{3} ; \mathbb{R}^{3}\right)$ the following identity holds:

$$
\int_{\mathbb{R}^{3}}|D A|^{2} \mathrm{~d} x=\int_{\mathbb{R}^{3}}\left\{|\nabla \times A|^{2}+(\operatorname{div} A)^{2}\right\} \mathrm{d} x .
$$

We point out on the other hand that the classical Sobolev embedding gives a constant $K>0$ so that, for $A \in$ $C_{0}^{\infty}\left(\mathbb{R}^{3} ; \mathbb{R}^{3}\right)$, it holds

$$
\left\{\int_{\mathbb{R}^{3}}|A|^{6} \mathrm{~d} x\right\}^{1 / 6} \leqslant K\left\{\int_{\mathbb{R}^{3}}|D A|^{2} \mathrm{~d} x\right\}^{1 / 2} .
$$

This in particular implies that either side of the identity (2.1) defines a norm in $C_{0}^{\infty}\left(\mathbb{R}^{3} ; \mathbb{R}^{3}\right)$. Denote then by $H$ the completion of $C_{0}^{\infty}\left(\mathbb{R}^{3} ; \mathbb{R}^{3}\right)$ with respect to the norm

$$
\|A\|_{H}=\left\{\int_{\mathbb{R}^{3}}\left\{|\nabla \times A|^{2}+(\operatorname{div} A)^{2}\right\} \mathrm{d} x\right\}^{1 / 2} .
$$

This makes $H$ a Hilbert space and

$$
H_{0}=\{A \in H: \operatorname{div} A=0\}
$$

a (strongly) closed subspace of $H$. It follows that $H_{0}$ is also weakly closed, since it is obviously convex. Furthermore, the norm that $H_{0}$ inherits from $H$ is equivalent in $H_{0}$ to the norm

$$
\|A\|_{0}=\left\{\int_{\mathbb{R}^{3}}|\nabla \times A|^{2} \mathrm{~d} x\right\}^{1 / 2}
$$


A similar construction could be carried out for $\Omega$ instead of $\mathbb{R}^{3}$ by means of the following lemma taken from [11]:

Lemma 2.2 [11]. Let $p \in] 1, \infty\left[\right.$. There is a constant $C=C(\Omega, p)$ such that for every $B \in W^{1, p}\left(\Omega ; \mathbb{R}^{3}\right)$ with $B \times v=0$ on $\partial \Omega$ we have

$$
\|B\|_{W^{1, p}} \leqslant C\left\{\int_{\Omega}\left(|\nabla \times B|^{p}+(\operatorname{div} B)^{p}\right) \mathrm{d} x\right\}^{1 / p},
$$

where $\|\cdot\|_{W^{1, p}}$ denotes the usual norm in $W^{1, p}\left(\Omega ; \mathbb{R}^{3}\right)$.

Remark 2.3. We denote

$$
W_{T}^{1, p}\left(\Omega ; \mathbb{R}^{3}\right)=\left\{B \in W^{1, p}\left(\Omega ; \mathbb{R}^{3}\right): B \times v=0 \text { on } \partial \Omega\right\} .
$$

Note that in this space

$$
\|B\|_{W_{T}^{1, p}}=\left\{\int_{\Omega}\left(|\nabla \times B|^{p}+(\operatorname{div} B)^{p}\right) \mathrm{d} x\right\}^{1 / p}
$$

is equivalent to the standard Sobolev norm. This is the classical Poincare inequality for this space. We also define analogous Hölder spaces,

$$
C_{T}^{0, \beta}\left(\Omega ; \mathbb{R}^{3}\right)=\left\{B \in C^{0, \alpha}\left(\Omega ; \mathbb{R}^{3}\right): B \times v=0 \text { on } \partial \Omega\right\}, \quad 0<\beta \leqslant 1 .
$$

In several instances we will make use of the Hodge decomposition for vector fields:

Lemma 2.4. There are constants $C_{1}, C_{2}=C_{1}(\Omega), C_{2}(\Omega)$ such that for any $A \in L^{2}\left(\Omega ; \mathbb{R}^{3}\right)$ one can find a pair $\left(\phi_{A}, B_{A}\right) \in W^{1,2}(\Omega) \times W_{T}^{1,2}\left(\Omega ; \mathbb{R}^{3}\right)$ satisfying

$$
\begin{aligned}
& A=\nabla \times B_{A}+\nabla \phi_{A} \quad \text { in } \Omega, \\
& \left\|\phi_{A}\right\|_{W^{1,2}}+\left\|B_{A}\right\|_{W_{T}^{1,2}} \leqslant C_{1}\|A\|_{L^{2}(\Omega)} \text { and } \\
& \operatorname{div}\left(B_{A}\right)=0 .
\end{aligned}
$$

The choice of $B_{A}$ is unique among divergence free vector fields and the choice of $\phi_{A}$ is unique among functions in $W^{1,2}(\Omega)$ with zero average. Moreover, when $A \in H$ one also has

$$
\left\|B_{A}\right\|_{W^{2,2}}+\left\|\phi_{A}\right\|_{W^{2,2}} \leqslant C_{2}\|A\|_{H} .
$$

Proof. This lemma is a special case of Lemma A.4 from [4], although similar forms of decomposition of vector fields have been derived much earlier (see Ladyzhenskaya [16] for one such version and some historical notes). We include in Section 7 a direct proof of this result in the case $\Omega=B_{R}(0) \subset \mathbb{R}^{3}$ for the reader's convenience.

\section{A solution to London's equation}

Next we minimize the magnetic energy using the tools derived in the previous section. The solution that we find can be thought of as an approximation to the magnetic field of the "Meissner state", since the magnetic energy, as defined in the introduction, is $F_{h_{\mathrm{ap}}}(A)=G_{\varepsilon}(1, A)$.

First, consider the case of a general given $h_{\mathrm{ap}}$, such that we can find $A_{\mathrm{ap}} \in W_{\text {loc }}^{2, \infty}\left(\mathbb{R}^{3} ; \mathbb{R}^{3}\right)$ with $\nabla \times A_{\text {ap }}=h_{\mathrm{ap}}$ and $\operatorname{div}\left(A_{\text {ap }}\right)=0$, in all of $\mathbb{R}^{3}$. By Lemma 2.4 there exists $B_{\text {ap }} \in W_{T}^{1,2}\left(\Omega ; \mathbb{R}^{3}\right)$, with $B_{\text {ap }} \times v=0$ on $\partial \Omega$ and

$$
A_{\text {ap }}=\nabla \times B_{\text {ap }}+\nabla \phi_{\text {ap }} \quad \text { in } \Omega .
$$

We then seek minimizers of the magnetic energy in the form, $A_{0}=A_{\text {ap }}+A_{1}$ with $A_{1} \in H_{0}$ minimizing

$$
F\left(A_{1}\right)=\frac{1}{2} \int_{\Omega}\left|\nabla \times\left(B_{A_{1}}+B_{\text {ap }}\right)\right|^{2} \mathrm{~d} x+\int_{\mathbb{R}^{3}}\left|\nabla \times A_{1}\right|^{2} \mathrm{~d} x .
$$


Here $B_{A_{1}}$ is determined by $A_{1}$ as in Lemma 2.4. We have the following existence theorem:

Theorem 3.1. The functional

$$
F\left(A_{1}\right)=\frac{1}{2} \int_{\Omega}\left|\nabla \times\left(B_{A_{1}}+B_{\text {ap }}\right)\right|^{2} \mathrm{~d} x+\int_{\mathbb{R}^{3}}\left|\nabla \times A_{1}\right|^{2} \mathrm{~d} x
$$

has a unique minimizer $A_{1} \in H_{0}$. Calling $A_{0}=A_{1}+A_{\mathrm{ap}}$, one has $A_{0} \in C_{\mathrm{loc}}^{1, \alpha}\left(\mathbb{R}^{3} ; \mathbb{R}^{3}\right)$. Moreover, $h_{0}=\nabla \times A_{0}$ is a weak solution of the system

$$
\left.\begin{array}{l}
\nabla \times h_{0}=\nabla \times h_{\text {ap }} \quad \text { in } \mathbb{R}^{3} \backslash \Omega, \\
h_{0}+\nabla \times \nabla \times h_{0}=\nabla \times \nabla \times h_{\text {ap }} \quad \text { and } \operatorname{div} h_{0}=0 \quad \text { in } \Omega, \\
h_{0}-h_{\text {ap }} \in L^{2}\left(\mathbb{R}^{3} ; \mathbb{R}^{3}\right), \quad \text { and } h_{0} \in C_{\text {loc }}^{0, \alpha}\left(\mathbb{R}^{3} ; \mathbb{R}^{3}\right) .
\end{array}\right\}
$$

Proof. First of all note that the functional is well defined, due to the uniqueness and continuity of $B_{A}$ as a function of $A \in H_{0}$ given by Lemma 2.2. Clearly the functional is continuous, coercive and strictly convex in $A \in H_{0}$. The existence and uniqueness of a minimizer of $F(A)$ in $H_{0}$ then follow immediately. Call the minimizer $A_{1}$. The critical point condition reads in this case

$$
\int_{\Omega} \nabla \times\left(B_{A_{1}}+B_{\mathrm{ap}}\right) \cdot \nabla \times B_{A} \mathrm{~d} x+\int_{\mathbb{R}^{3}} \nabla \times A_{1} \cdot \nabla \times A \mathrm{~d} x=0
$$

for all $A \in H_{0}$. The boundary conditions of $B_{A}$ imply that for any function $\phi \in W^{1,2}(\Omega)$

$$
\int_{\Omega} \nabla \times\left(B_{A_{1}}+B_{\mathrm{ap}}\right) \cdot \nabla \phi \mathrm{d} x=0
$$

so the critical point condition can be rewritten as

$$
\begin{gathered}
\int_{\Omega} \nabla \times\left(B_{A_{1}}+B_{\text {ap }}\right) \cdot\left(\nabla \phi_{A}+\nabla \times B_{A}\right) \mathrm{d} x+\int_{\mathbb{R}^{3}} \nabla \times A_{1} \cdot \nabla \times A \mathrm{~d} x \\
=\int_{\Omega} \nabla \times\left(B_{A_{1}}+B_{\text {ap }}\right) \cdot A \mathrm{~d} x+\int_{\mathbb{R}^{3}} \nabla \times A_{1} \cdot \nabla \times A \mathrm{~d} x=0 .
\end{gathered}
$$

Integrating the last integral in this expression by parts one obtains

$$
\int_{\mathbb{R}^{3}} A \cdot\left(\nabla \times \nabla \times A_{1}+\chi_{\Omega} \nabla \times\left(B_{A_{1}}+B_{\text {ap }}\right)\right) \mathrm{d} x+\int_{\partial \Omega}\left(\left[\nabla \times A_{1}\right] \times v\right) \cdot A \mathrm{~d} S=0 .
$$

Here $\chi_{\Omega}$ represents the characteristic function of $\Omega$, and $\left[\nabla \times A_{1}\right]$ represents the jump of $\nabla \times A_{1}$ across the border $\partial \Omega$. We conclude that

$$
\nabla \times \nabla \times A_{1}+\chi_{\Omega} \nabla \times\left(B_{1}+B_{\text {ap }}\right)=0
$$

a.e. in $\mathbb{R}^{3}$, and $\left[\nabla \times A_{1}\right] \times v=0$ on $\partial \Omega$. Replacing $\nabla \times B_{1}=A_{1}-\nabla \phi_{1}$ in this last expression we obtain

$$
\nabla \times \nabla \times A_{1}+\chi_{\Omega} A_{1}=\chi_{\Omega}\left(-\nabla \times B_{\text {ap }}+\nabla \phi_{1}\right) .
$$

The fact that $\operatorname{div} A_{1}=0$ yields now

$$
-\Delta A_{1}+\chi_{\Omega} A_{1}=\chi_{\Omega}\left(-\nabla \times B_{\text {ap }}+\nabla \phi_{1}\right) .
$$

Since $A \in H_{0},(2.10)$ yields immediately $\nabla \phi_{1} \in W^{1,2}\left(\Omega ; \mathbb{R}^{3}\right)$, and the same holds for $\nabla \times B_{\text {ap }}$. The classical Sobolev embedding gives that the right-hand side of (3.3) is in $L^{p}\left(\Omega ; \mathbb{R}^{3}\right)$ for $1 \leqslant p \leqslant 6$. We fix $3<p \leqslant 6$ and appeal to [8], Corollary 8.36 and the remark right after to claim $A_{1} \in C_{\text {loc }}^{1, \alpha}\left(\mathbb{R}^{3}, \mathbb{R}^{3}\right)$ for $0<\alpha<1-n / p$.

Finally, $A_{0}=A_{1}+A_{\text {ap }}$ and $h_{0}=\nabla \times A_{0}$, so the conclusions of the theorem regarding the equations and the regularity of $h_{0}$ hold. 
Now we specialize to the case which we require for our analysis of vortices. We assume $\Omega=B_{R}(0)$, and

$$
h_{\text {ap }}=\hat{e}_{3} \text {. }
$$

We then choose (for example) $A_{\text {ap }}=A_{\text {ap }}^{*}=\left(-\frac{y}{2}, \frac{x}{2}, 0\right)$, and obtain by Theorem 3.1 a minimizer $A_{1}^{*} \in H_{0}, A^{*}=$ $A_{\mathrm{ap}}^{*}+A_{1}^{*}, h^{*}=\nabla \times A^{*}$, and from Lemma 2.4 a corresponding $B^{*}, \phi^{*}$. These may be calculated explicitly in spherical coordinates; see [18]. Let $r=\sqrt{x_{1}^{2}+x_{2}^{2}+x_{3}^{2}}, \theta \in[0, \pi]$ measure the angle down from the north pole of the unit sphere, and $\phi \in[0,2 \pi)$ the equatorial angle measured from the $x_{1}$-axis. We obtain:

$$
h^{*}=\frac{3 R}{r^{2} \sinh R}\left(\cosh r-\frac{\sinh r}{r}\right) \cos \theta \hat{r}+\frac{3 R}{2 r^{2} \sinh R}\left(\cosh r-\frac{1+r^{2}}{r} \sinh r\right) \sin \theta \hat{\theta} .
$$

Finally, writing

$$
A^{*}=\nabla \times B^{*}+\nabla \phi^{*}
$$

as in Lemma $2.4, B^{*}$ can be expressed as

$$
B^{*}=-h^{*}-c^{*} \hat{e}_{3}, \quad \text { with } c^{*}=\frac{3}{2 R \sinh R}\left(\cosh R-\frac{1+R^{2}}{R} \sinh R\right) .
$$

In case $h_{\mathrm{ap}}=\Lambda \hat{e}_{3}$ for constant $\Lambda$, we note that by homogeneity the corresponding minimizer of the magnetic energy is given simply by $A_{0}=\Lambda A^{*}, h_{0}=\Lambda h^{*}, B_{0}=\Lambda B^{*}, \phi_{0}=\Lambda \phi^{*}$.

\section{The limiting energy}

In this section we study the limiting energy of vortices, sometimes called the "line energy", obtained formally in the limit $\varepsilon \rightarrow 0$ as in the introduction. Let us recall here that the energy of a vortex line $T$ (an integer multiplicity rectifiable 1-current with $\partial T=0)$, may be written as

$$
\mathcal{G}_{\lambda}(T)=M(T)-2 \lambda T\left(B^{*}\right),
$$

where $M(T)$ is the mass of the current $T, T\left(B^{*}\right)$ gives its action on the vector field $B^{*}$, and $B^{*}$ comes from (3.6). If we knew that the vortex line $T$ were actually an oriented curve lying in a two-dimensional cross-section

$$
B_{R}^{2}:=\left\{x=\left(x_{1}, x_{2}, x_{3}\right): x_{1}^{2}+x_{3}^{2}<R^{2}, x_{2}=0\right\},
$$

of the sphere, then we may express the limiting line energy in more classical terms as

$$
\mathcal{G}_{\lambda}(T)=\int_{T} \mathrm{~d} s-2 \lambda \int_{T} B^{*} \cdot \tau \mathrm{d} s,
$$

where $\tau$ is the unit tangent to the curve $T$. Since $\partial T=\emptyset, T$ partitions $B_{R}^{2}$ into two domains, each with boundary consisting of $T$ together perhaps with some piece of $\partial B_{R}^{2}$, properly oriented. We choose the domain $D_{T}$ to be the one for which the positively oriented normal vector is $\vec{n}_{D_{T}}=\hat{e}_{2}$. (For example, if $T$ is the vertical diameter oriented upwards, $D_{T}$ lies to the right of $T$.) Since $B^{*} \cdot \tau=0$ on $\partial B_{R}^{2}$, we may interpret the second line integral as being over the closed curve $\partial D_{T}$, and applying Green's Theorem, we obtain an equivalent form of the line energy,

$$
\mathcal{G}_{\lambda}(T)=\int_{T} \mathrm{~d} s-2 \lambda \int_{D_{T}} \nabla \times B^{*} \cdot \hat{e}_{2} \mathrm{~d} x_{1} \mathrm{~d} x_{3} .
$$

We point out that spherical coordinates in $\mathbb{R}^{3}$ restricted to the cross-section $B_{R}^{2}$ gives a system of polar coordinates $(r, \theta)$, if we now permit the angle $\theta \in[-\pi, \pi]$, where we recall that $\theta=0$ corresponds to the positive $x_{3}$-axis. With this understanding, the integrand in the second term of $\mathcal{G}_{\lambda}$ has the form:

$$
\nabla \times B^{*} \cdot \hat{e}_{2}=\frac{3 R}{2 \sinh R}\left(\cosh r-\frac{\sinh r}{r}\right) \frac{\sin \theta}{r}=: f(r) \frac{\sin \theta}{r} .
$$

Unfortunately we cannot show that the limiting current associated to global minimizers of the Ginzburg-Landau energy is indeed a single curve, or even that it is rectifiable (and thus morally equivalent to a union of Lipschitz 
curves.) In fact, looking at the 2-d situation as described by Sandier and Serfaty in [24,23] it is reasonable to expect for $\left|h_{\mathrm{ap}}^{\varepsilon}\right|>H_{c_{1}}=\mathrm{O}(|\ln \varepsilon|)$ and global minimizers $\left(u_{\varepsilon}, A_{\varepsilon}\right)$ of $(1.1)$ that $E_{\varepsilon}\left(u_{\varepsilon}\right)=\mathrm{O}\left(|\ln \varepsilon|^{2}\right)$ and it is known that in this case the Jacobians $J\left(u_{\varepsilon}\right) /|\ln \varepsilon|$ converge but not necessarily to a rectifiable limit.

Another source of difficulties is the fact that even among integer multiplicity rectifiable 1-currents, the limiting energy we consider either has a trivial 'vortexless' global minimizer $(T=0)$ or is unbounded from below. Indeed, if we had an oriented curve $T$ with $\mathcal{G}_{\lambda}(T)<0$, then by superimposing $n$ copies of $T$ (perhaps with rotations to make them distinct) we obtain a new current with energy $n$ times $\mathcal{G}_{\lambda}(T)$. This clearly implies that $\mathcal{G}_{\lambda}$ is unbounded below.

To circumvent these difficulties we employ two different approaches. First, we can still find a "global minimizer" of the line energy if we restrict our attention to single multiplicity vortex lines. This approach will enable us to identify a candidate for the "lower critical field", the value of $h_{\text {ap }}$ at which vortices first become energetically feasible. Second, with an eye in building local minimizers to the Ginzburg-Landau energy $G_{\varepsilon}$ given by (1.1), we show that the diameter is an isolated local minimizer of the line energy in an appropriate topology among integer multiplicity rectifiable 1-currents when $h_{\text {ap }}$ is large enough. This result will follow from a construction similar to that of $[19,13]$.

\subsection{Global minimizers of the line energy}

We follow [1] and pose the line energy problem in the context of Cacciopoli sets. The limiting problem being posed in the two-dimensional cross-section $B_{R}^{2}$, we may identify the curve $T$ with its associated domain $D_{T}$, and use $\chi=\chi_{D_{T}}$, the characteristic function of $D_{T}$, as the variable. In this context, $\mathcal{G}_{\lambda}(T)=\widetilde{\mathcal{G}}_{\lambda}\left(\chi_{D_{T}}\right)$, with

$$
\widetilde{\mathcal{G}}_{\lambda}(\chi)=|\nabla \chi|\left(B_{R}^{2}\right)-\int_{B_{R}^{2}} 2 \lambda f(r) \sin \theta \chi \mathrm{d} r \mathrm{~d} \theta,
$$

defined for $\chi \in \mathcal{B}:=\mathrm{BV}\left(B_{R}^{2} ;\{0,1\}\right)$. (We recall the definition of $f(r)$ from (4.3).)

Lemma 4.1. For any $\lambda \geqslant 0$ :

(i) there exists a minimizer $\chi_{*}$ of $\widetilde{\mathcal{G}}_{\lambda}$ in $\mathcal{B}$ which is symmetric with respect to reflection in the $x_{1}$-axis;

(ii) supp $\chi_{*} \subset \overline{B_{R}^{2+}}$ is supported in the right half-disk;

(iii) $\partial\left(\operatorname{supp} \chi_{*}\right)$ consists of a single analytic curve meeting the boundary $\partial B_{R}^{2}$ at right angles.

We remark that by Theorem 1.3 of [1], the minimizer $\chi_{*}$ is unique for almost every $\lambda>0$.

Proof. The existence of a minimizer $\chi_{*}=\chi_{E_{*}}$ follows from general properties of BV functions. To prove symmetry in the $x_{1}$-axis, if

$$
\left.\int_{B_{R}^{2} \cap\left\{x_{3}>0\right\}}\left(\left|\nabla \chi_{*}\right|-2 \lambda f(r) \sin \theta\right) r \mathrm{~d} r \mathrm{~d} \theta<\int_{B_{R}^{2} \cap\left\{\chi_{3}<0\right\}}\left(\left|\nabla \chi_{*}\right|-2 \lambda f(r) \sin \theta\right)\right) r \mathrm{~d} r \mathrm{~d} \theta,
$$

define

$$
\chi_{* *}\left(x_{1}, x_{3}\right)= \begin{cases}\chi_{*}\left(x_{1}, x_{3}\right), & \text { if } x_{3} \geqslant 0 \\ \chi_{*}\left(x_{1},-x_{3}\right), & \text { if } x_{3}<0\end{cases}
$$

Then by the symmetry of the integrands we would have $\widetilde{\mathcal{G}_{\lambda}}\left(\chi_{* *}\right) \leqslant \widetilde{\mathcal{G}_{\lambda}}\left(\chi_{*}\right)$, and thus we may assume that $\chi_{*}$ is symmetric.

To obtain regularity, we also argue as in [1]. For any $\chi \in \mathcal{B}$ let

$$
F(\chi):=\int_{B_{R}^{2}} f(r) \sin \theta \chi \mathrm{d} r \mathrm{~d} \theta
$$

and define $\ell_{*}=F\left(\chi_{*}\right)$ where $\chi_{*}$ is a minimizer of $\widetilde{\mathcal{G}}_{\lambda}$. Then $\chi_{*}$ also attains the absolute minimum of the perimeter functional $P(\chi)=\int_{B_{R}^{2}}|\nabla \chi|$ under the (weighted) area constraint $F(\chi)=\ell_{*}$. The regularity of $\partial E_{*}$ then follows from 
the regularity of minimal surfaces in low dimensions [10]. It implies that $E_{*}=\operatorname{supp} \chi_{*}$ consists of countably many relatively closed components, each bounded either by a closed analytic curve or by a countable number of analytic arcs meeting the boundary at right angles. Call these components $\left\{E_{k}=\operatorname{supp} \chi_{k}\right\}$, so that $\chi_{*}=\sum_{k} \chi_{k}$.

First we show that there are only finitely many such components. Let $\delta>0$ be fixed, to be chosen later. Since

$$
\int_{B_{R}^{2}}|\nabla \chi|=\sum_{k=1}^{\infty} \int_{B_{R}^{2}}\left|\nabla \chi_{k}\right|<\infty,
$$

for all $k \geqslant K$ sufficiently large $\int_{B_{R}^{2}}\left|\nabla \chi_{k}\right|<\delta$. From the relative isoperimetric inequality (see Theorem 5.4.3 of [27]) we conclude

$$
\int_{B_{R}^{2}} \frac{f(r)}{r} \sin \theta \chi_{k} r \mathrm{~d} r \mathrm{~d} \theta \leqslant C \int_{B_{R}^{2}} \chi_{k} \mathrm{~d} x \leqslant C^{\prime} \delta^{2},
$$

with constant $C^{\prime}$ independent of $k, \delta$. Now fix $\delta$ small enough (depending on $\lambda$ ) so that $\delta-2 \lambda C^{\prime} \delta^{2}>0$. Then, $\widetilde{\mathcal{G}}_{\lambda}\left(\chi_{k}\right)>0$ for all $k \geqslant K$, and hence

$$
\widetilde{\mathcal{G}_{\lambda}}\left(\sum_{k=1}^{K} \chi_{k}\right)=\sum_{k=1}^{K} \widetilde{\mathcal{G}_{\lambda}}\left(\chi_{k}\right)<\widetilde{\mathcal{G}_{\lambda}}\left(\chi_{*}\right),
$$

a contradiction unless $\chi_{*}$ only had finitely many connected components.

Now we will show that $E_{*}=\operatorname{supp} \chi_{*} \subset \overline{B_{R}^{2+}}$. Let $\widetilde{E}_{*}=\left\{\left(x_{1}, x_{3}\right) \mid\left(-x_{1}, x_{3}\right) \in E_{*}\right\}$ be its reflection with respect to the $x_{3}$-axis. First we claim that if $\left|\nabla \chi_{*}\right|\left(\left\{x_{1}=0\right\}\right)>0$, then $E_{*}=\overline{B_{R}^{2+}}$, the entire right half-disk. Indeed, assume there exists a point $P \in\left\{x_{1}=0\right\}$ in the support of $\left|\nabla \chi_{E_{*}}\right|$. By regularity, near this point $\partial E_{*}$ consists of a smooth curve, and the measure $\left|\nabla \chi_{E_{*}}\right|$ coincides locally with arclength measure on that curve. In particular, the curve must lie along some interval of the diameter $\left\{x_{1}=0\right\}$, and can be represented as a graph $x_{1}=\gamma\left(x_{3}\right)$ in some larger interval on the $x_{3}$-axis. This curve then satisfies the Euler-Lagrange equations for the limit energy $\mathcal{G}_{\lambda}$,

$$
\frac{\gamma^{\prime \prime}}{\left(1+\left(\gamma^{\prime}\right)^{2}\right)^{3 / 2}}=\frac{3 R \lambda}{2 \sinh (R) r_{\gamma}^{2}}\left(\cosh r_{\gamma}-\frac{\sinh r_{\gamma}}{r_{\gamma}}\right) \gamma\left(x_{3}\right),
$$

where $r_{\gamma}=\left(\gamma\left(x_{3}\right)^{2}+x_{3}^{2}\right)^{1 / 2}$. By ODE uniqueness (note that the right-hand side is smooth at $r_{\gamma}=0$ ) the curve must coincide with the diameter $\gamma\left(x_{3}\right) \equiv 0$. We conclude that $E_{*}=B_{R}^{2+}$, the entire half-disk, since it gives the largest value of $F(\chi)$ and the smallest possible total perimeter given that $\partial E_{*}$ contains the diameter $S_{1}$.

Next assume that $E_{*}$ is not the entire half-disk (and so $\left|\nabla \chi_{*}\right|\left(\left\{x_{1}=0\right\}\right)=0$,) and consider the symmetric difference $E_{*} \Delta \widetilde{E}_{*}$. Since

$$
\chi_{E_{*} \Delta \widetilde{E}_{*}}(x)=\chi_{E_{*} \cup \widetilde{E}_{*}}(x)-\chi_{E_{*} \cap \widetilde{E}_{*}}(x),
$$

we apply Lemma 2.2 of [1] to conclude that

$$
\left|\nabla \chi_{E_{*} \Delta \widetilde{E}_{*}}\right|=\left|\nabla\left(\chi_{E_{*} \cup \widetilde{E}_{*}}-\chi_{E_{*} \cap \tilde{E}_{*}}\right)\right| \leqslant\left|\nabla \chi_{E_{*} \cup \widetilde{E}_{*}}\right|+\left|\nabla \chi_{E_{*} \cap \widetilde{E}_{*}}\right| \leqslant\left|\nabla \chi_{E_{*}}\right|+\left|\nabla \chi_{\widetilde{E}_{*}}\right|=2\left|\nabla \chi_{E_{*}}\right|,
$$

by symmetry. Now $E_{*} \Delta \widetilde{E}_{*}$ is a disjoint union of two components, $E_{*} \Delta \widetilde{E}_{*}=F^{+} \cup F^{-}$, with $F^{+}$supported in the right half-disk, and $F^{-}$supported in the left half-disk, and one is the reflection of the other in the $x_{3}$-axis, $F^{-}=\widetilde{F}^{+}$. Note that $\left|\nabla \chi_{F^{+}}\right|$defines a measure supported in the closed half-disk $\overline{B_{R}^{2+}}$, and similarly for $\left|\nabla \chi_{F^{-}}\right|$, supported in $\overline{B_{R}^{2-}}$. By the preceding paragraph, $\left|\nabla \chi_{E_{*}}\right|\left(S_{1}\right)=\left|\nabla \chi_{\widetilde{E}_{*}}\right|\left(S_{1}\right)=0$, so by (4.6), $\left|\nabla \chi_{E_{*} \Delta \widetilde{E}_{*}}\right|\left(S_{1}\right)=0$ as well. Hence we conclude that the measures $\left|\nabla \chi_{F^{+}}\right|,\left|\nabla \chi_{F^{-}}\right|$are mutually singular, supported in the open half-disks $B_{R}^{2+}, B_{R}^{2-}$. By symmetry of the reflection, the total mass satisfies:

$$
\left|\nabla \chi_{E_{*} \Delta \widetilde{E}_{*}}\right|\left(B_{R}^{2}(0)\right)=\left|\nabla \chi_{F^{+}}\right|\left(B_{R}^{2}(0)\right)+\left|\nabla \chi_{F^{-}}\right|\left(B_{R}^{2}(0)\right)=2\left|\nabla \chi_{F^{+}}\right|\left(B_{R}^{2}(0)\right) .
$$

If we then choose $\chi_{* *}=\chi_{F^{+}}$, then $\chi_{* *}$ has support in $B_{R}^{2+}$ and by (4.6),

$$
\left|\nabla \chi_{* *}\right|\left(B_{R}^{2}(0)\right) \leqslant\left|\nabla \chi_{*}\right|\left(B_{R}^{2}(0)\right) .
$$


We now claim that $F\left(\chi_{* *}\right)=F\left(\chi_{*}\right)$, and hence the total energy $\widetilde{\mathcal{G}}_{\lambda}\left(\chi_{* *}\right) \leqslant \widetilde{\mathcal{G}}_{\lambda}\left(\chi_{*}\right)$. Indeed, the original set $E_{*}=$ $F^{+} \cup\left[E_{*} \cap \widetilde{E}_{*}\right]$. The set $E_{*} \cap \widetilde{E}_{*}$ being symmetric with respect to the $x_{3}$-axis and the integrand of $F$ being odd, this part integrates to zero and we obtain the desired identity. This completes the proof of part (ii) of Lemma 4.1.

We now claim that each component $E_{k}=\operatorname{supp} \chi_{k}$ of $E_{*}$ must either be bounded by a single, closed curve inside $B_{R}^{2+}$ or be as in (iii). Indeed, if supp $\chi_{k}$ has interior boundaries, we obtain a set whose characteristic function has smaller energy by eliminating the interior boundaries, since the arclength is reduced and the integrand of $F$ is positive in $B_{R}^{2+}$. The same remark applies if supp $\chi_{k}$ contacts the boundary and has several boundary arcs contacting $\partial B_{R}^{2+}$. Since the minimizer is contained in the half-disk each connected component $E_{k}$ which contacts $\partial B_{R}^{2}$ must be enclosed by a single curve $C_{1}$ from $\partial E_{k}$ connecting the boundary at two extreme angles $0 \leqslant \theta_{1}<\theta_{2} \leqslant \pi$, together with the corresponding arc $C_{2}$ along the half-circle $\partial B_{R}^{2+}$. Denote the simple region enclosed by $C_{1}, C_{2}$ in $B_{R}^{2+}$ by $E_{k}^{\prime}$. Since the integrand of $F$ is positive and $C_{1} \subset \partial E_{k}, \widetilde{\mathcal{G}}_{\lambda}\left(\chi_{E_{k}^{\prime}}\right) \leqslant \widetilde{\mathcal{G}}_{\lambda}\left(\chi_{k}\right)$, which proves the claim.

Next we observe that the energy behaves in a simple, monotone way if we translate the connected components $\chi_{k}$ or rotate them along the boundary of the half-disk. Namely, the perimeter is unchanged by each of these displacements and the magnetic term increases as we increase $x_{1}=r \sin \theta$. Suppose that one of the $\chi_{k}$ is supported entirely in the interior of $B_{R}^{2+}$. By translation to the right we decrease the energy $\widetilde{\mathcal{G}}_{\lambda}$. This may be done until either the component meets the boundary $\partial B_{R}^{2+}$ or until the first contact of the support of $\chi_{k}$ with some other component of supp $\chi_{*}$. This second possibility is impossible, since the analyticity of the boundary arcs precludes their intersection inside $B_{R}^{2}$. Hence each component of supp $\chi_{*}$ must contact the boundary of the half-disk. By rotating each component along the boundary in the direction of increasing $x_{1}=r \sin \theta$ we again decrease $\widetilde{\mathcal{G}}_{\lambda}$, and hence these components may be assumed to be pairwise in contact with one another along $\partial B_{R}^{2+}$. We now show that this situation cannot occur either. Indeed, suppose that $\gamma_{1}$ and $\gamma_{2}$ are boundary arcs corresponding to two components of supp $\chi_{*}$ which meet at the same boundary point $P \in \partial B_{R}^{2+}$. By the above arguments, these curves do not touch inside $B_{R}^{2+}$ and each meets $\partial B_{R}^{2+}$ normally. Therefore, there must exist points $P_{1} \in \gamma_{1}$ and $P_{2} \in \gamma_{2}$ so that the line segment $\overline{P_{1} P_{2}}$ joining them intersects no other component of $\chi_{*}$. Clearly, $\overline{P_{1} P_{2}}$ is shorter than the arcs connecting $P_{1}$ to $P_{2}$ via the boundary point $P$. Thus, if we replace the portion of the arcs $\gamma_{1}, \gamma_{2}$ between $P_{1}, P_{2}$ and $\partial B_{R}^{2+}$ by this segment, we obtain a Cacciopoli set whose characteristic function would have smaller energy $\widetilde{\mathcal{G}}_{\lambda}$.

We remark here that the conclusions of Lemma 4.1 hold for problems in $\mathrm{BV}(\omega ;\{0,1\})$ for general symmetric twodimensional domains $\omega$ other than the disk, provided that the integrand $f(r) \sin \theta$ is replaced by a function having the appropriate symmetry and monotonicity properties used in proving the lemma.

The following result completely classifies the global minimizers of the functional $\widetilde{\mathcal{G}}_{\lambda}$ in terms of the field strength parameter $\lambda$. The result is strongly dependent on the superconducting domain being a ball.

Proposition 4.2. Let $f(r)$ be defined as in (4.3), and set

$$
\lambda^{*}=\frac{R}{2 \int_{0}^{R} f(r) \mathrm{d} r}=\frac{\sinh R}{3 \int_{0}^{R}(\cosh r-(\sinh r) / r) \mathrm{d} r} .
$$

- If $0 \leqslant \lambda<\lambda^{*}$ then the global minimizer of $\widetilde{\mathcal{G}_{\lambda}}$ is the vortex free configuration, $\chi \equiv 0$.

- If $\lambda>\lambda^{*}$ then $\widetilde{\mathcal{G}}_{\lambda}$ is minimized by $\chi=\chi_{B_{R}^{2+}}$, corresponding to the vortex along the vertical diameter $S_{1}$.

Proof. The key observation is that $\widetilde{\mathcal{G}}_{\lambda}(0)=0$, and so the global minimizer is a vortex configuration if and only if $\min _{\mathcal{B}} \widetilde{\mathcal{G}}_{\lambda}<0$. We begin by noting that the energy of the diameter vortex is

$$
\widetilde{\mathcal{G}}_{\lambda}\left(\chi_{B_{r}^{2+}}\right)=2 R-4 \lambda \int_{0}^{R} f(r) \mathrm{d} r
$$

and hence $\widetilde{\mathcal{G}}_{\lambda}\left(\chi_{B_{R}^{2+}}\right)<0=\widetilde{\mathcal{G}}_{\lambda}(0)$ exactly when $\lambda<\lambda^{*}$.

Next, assume that $\chi_{*} \not \equiv 0$ is a symmetric global minimizer with support in $B_{R}^{2+}$. We claim that there is an angle $\theta_{0} \in[0, \pi / 2)$ such that

$$
E_{*}=\operatorname{supp} \chi_{*} \subset \Sigma_{\theta_{0}}:=\left\{(r, \theta): \theta_{0} \leqslant \theta \leqslant \pi-\theta_{0}, 0 \leqslant r \leqslant R\right\},
$$


and such that $\partial E_{*}$ meets $\partial B_{R}^{2+}$ at angle $\theta_{0}$ (and at $\pi-\theta_{0}$.) Indeed, define $\theta_{0}$ to be the infimum of all angles $\theta$ for which the ray intersects $E_{*}$. Then by the symmetry of the minimizer (4.7) is satisfied. Since $E_{*}$ is closed, the infimum is attained, the optimum ray intersecting $E_{*}$ either on $\partial B_{R}^{2+}$ (in which case the claim is proven) or at some interior point $P$ lying on a boundary arc of $\partial E_{*}$. Since the ray is a radius of the circle, the segment attaching $P$ to $\partial B_{R}^{2+}$ along that radius has shorter length than the piece of arc forming part of the boundary of supp $\chi_{k}$. By replacing that arc (connecting $P$ to $\partial B_{R}^{2+}$ ) with the radial segment we would therefore create a new Cacciopoli set with smaller energy than $\chi_{*}$, and hence this case is impossible and the claim must hold.

We are now ready to prove the first assertion of the proposition. Assume $0 \leqslant \lambda<\lambda^{*}$. Since the boundary of supp $\chi_{*}$ originates at $\partial B_{R}^{2+}$ at angles $\theta_{0}, \pi-\theta_{0}$, the total perimeter is at least

$$
\int_{B_{R}^{2}}\left|\nabla \chi_{*}\right| \geqslant 2 R \cos \theta_{0}
$$

On the other hand we estimate the magnetic energy from above by comparing with the sector $\Sigma_{\theta_{0}}$,

$$
\int_{B_{R}^{2}} f(r, \theta) \chi_{*} \mathrm{~d} r \mathrm{~d} \theta \leqslant \int_{\Sigma_{\theta_{0}}} f(r, \theta) \mathrm{d} r \mathrm{~d} \theta=\int_{0}^{R} \int_{\theta_{0}}^{\pi-\theta_{0}} \sin \theta f(r) \mathrm{d} \theta \mathrm{d} r=2 \cos \theta_{0} \int_{0}^{R} f(r) \mathrm{d} r .
$$

Together, we have the lower bound on the energy,

$$
\widetilde{\mathcal{G}}_{\lambda}\left(\chi_{*}\right) \geqslant 2\left(R-2 \lambda \int_{0}^{R} f(r) \mathrm{d} r\right) \cos \theta_{0}>0
$$

when $0 \leqslant \lambda<\lambda^{*}$. Therefore, in this range of $\lambda$ the global minimizer must be the vortexless configuration.

Now consider the case $\lambda>\lambda^{*}$. We already know from the first paragraph of the proof that in this regime the diameter has negative energy, and hence the global minimizer is a vortex solution. Suppose $\chi_{*}$ is a symmetric global minimizer. By Lemma 4.1 its interior boundary is a single analytic curve, $\gamma$. Let $D_{\gamma}$ be the region in $B_{R}^{2+}$ bounded by $\gamma$ and the diameter $S_{1}$, and the circular $\operatorname{arcs} C_{1}=\left\{(R, \theta): 0 \leqslant \theta \leqslant \theta_{0}\right\}$ and $C_{2}=\left\{(R, \theta): \pi-\theta_{0} \leqslant \theta \leqslant \pi\right\}$. We use the following simple estimation:

$$
\begin{aligned}
0 & =\int_{D_{\gamma}} \Delta x_{1}=\int_{\partial D_{\gamma}} \frac{\partial x_{1}}{\partial v} \mathrm{~d} s=-\int_{S_{1}} \mathrm{~d} s+\int_{\gamma} \nu_{1} \mathrm{~d} s+\int_{0}^{\theta_{0}} R \sin \theta \mathrm{d} \theta+\int_{\pi-\theta_{0}}^{\pi} R \sin \theta \mathrm{d} \theta \\
& \leqslant-2 R+\ell(\gamma)+2 R\left(1-\cos \theta_{0}\right),
\end{aligned}
$$

where $\ell(\gamma)$ denotes the arclength of $\gamma$ and $v_{1}=v \cdot \hat{e}_{1}$.

Recall from the previous part that supp $\chi_{*} \subset \Sigma_{\theta_{0}}$ for some sector of angle $\theta_{0} \geqslant 0$. Note that in case $\theta_{0}=0$ from the previous paragraph we must have $\ell(\gamma) \geqslant 2 R$. Since $F\left(\chi_{*}\right) \leqslant F\left(\chi_{B_{R}^{2+}}\right)$ is optimized by the entire half-disk, we conclude that $\widetilde{\mathcal{G}}_{\lambda}\left(\chi_{*}\right) \geqslant \widetilde{\mathcal{G}}_{\lambda}\left(\chi_{B_{R}^{2+}}\right)$ in this case, and so $\theta_{0}=0$ can only occur when the vortex lies along the diameter $S_{1}$. For a global minimizer $\chi_{*} \neq S_{1}$ then we assume $\theta_{0}>0$. In this situation, since supp $\chi_{*} \subset \Sigma_{\theta_{0}}$, we must have

$$
D_{\gamma} \supset \Delta_{\theta_{0}}:=\left\{(r, \theta): 0 \leqslant r \leqslant R, 0 \leqslant \theta \leqslant \theta_{0} \text { or } \pi-\theta_{0} \leqslant \theta \leqslant \pi\right\} .
$$

In this way we obtain the following lower bound on the magnetic term,

$$
\int_{B_{R}^{2}} f(r) \sin \theta \chi_{*} \mathrm{~d} r \mathrm{~d} \theta \geqslant \int_{\Delta_{\theta_{0}}} f(r) \sin \theta \mathrm{d} r \mathrm{~d} \theta=2 \int_{0}^{R} \int_{0}^{\theta_{0}} f(r) \sin \theta \mathrm{d} r \mathrm{~d} \theta=2\left(1-\cos \theta_{0}\right) \int_{0}^{R} f(r) \mathrm{d} r .
$$

In particular, the above inequality together with (4.8) yields

$$
\mathcal{G}_{\lambda}(\gamma)-\mathcal{G}_{\lambda}\left(S_{1}\right)=\ell(\gamma)-2 R+2 \lambda \int_{D_{\gamma}} f(r) \sin \theta \mathrm{d} r \mathrm{~d} \theta \geqslant 2\left(1-\cos \theta_{0}\right)\left(2 \lambda \int_{0}^{R} f(r) \mathrm{d} r-R\right)>0
$$


when $\lambda>\lambda^{*}$ (recall $\theta_{0}>0$ ) contradicting that $\chi_{*}$ is a global minimizer. In other words, the unique global minimizer is the diameter $S_{1}$.

\subsection{The diameter as a local minimizer}

In this part we adopt a different point of view and treat the vortices as they more naturally occur in the $\varepsilon \rightarrow 0$ limit process, that is as integer multiplicity rectifiable 1-currents. This approach has two advantages: we can use it to construct local minimizers of the $\varepsilon$-problem by employing the $\Gamma$-convergence trick of Kohn and Sternberg [15] as in $[19,13]$, and we can consider arbitrary degree $n$ for $S_{n}$, the limiting vorticity.

We seek local minimizers of the line energy, "local" being measured by the norm dual to the standard Hölder norm for vector fields, this is, for $T \in \mathcal{R}_{1}(\Omega)$ we define

$$
\|T\|_{0,1}^{*}=\sup _{\left\{B \in C_{T}^{0,1}\left(\Omega ; \mathbb{R}^{3}\right):\|B\|_{C^{0,1}} \leqslant 1\right\}} T(B) .
$$

Here $\|\cdot\|_{C^{0,1}}$ represents the usual Hölder norm, and

$$
C_{T}^{0,1}\left(\Omega ; \mathbb{R}^{3}\right)=\left\{B \in C^{0,1}\left(\Omega ; \mathbb{R}^{3}\right): B \times v=0 \text { on } \partial \Omega\right\} .
$$

We will make use of the fact that $T \in \mathcal{R}_{1}(\Omega)$ can always be represented as

$$
T(B)=\int_{\Gamma} n(x) B \cdot \tau \mathrm{d} H_{(1)} .
$$

Here $\Gamma \subset \Omega$ is a 1-rectifiable set, that is, $\Gamma=\Gamma_{0} \bigcup_{n \geqslant 1} \Gamma_{k}$ where $H^{(1)}\left(\Gamma_{0}\right)=0$ and for each $k \geqslant 1$ there is a set $I_{k} \subset \mathbb{R}$ and a Lipschitz function $f_{k}: \mathbb{R} \rightarrow \mathbb{R}^{3}$ with $\Gamma_{k}=f_{k}\left(I_{k}\right)$. Also, the functions $n: \Gamma \rightarrow \mathbb{Z}$ and $\tau: \Gamma \rightarrow \mathbb{R}^{3}$ are assumed to be $H^{(1)}$ measurable, and $|\tau|=1 H^{(1)}$ a.e. By the support of $T$ we always refer to the set $\Gamma$. Note that this set $\Gamma$ is only defined up to a set of $H^{(1)}$-measure 0 . Finally, we will denote by $B_{R}^{2}$ a ball around 0 in $\mathbb{R}^{2}$ rather than $\mathbb{R}^{3}$, for which we reserve the notation $B_{R}(0)$. We will also write $B_{R}^{2,+} \equiv\left\{\left(x_{1}, x_{3}\right) \in B_{R}^{2}: x_{1} \geqslant 0\right\}$.

The main result of this section is

Theorem 4.3. Let $\lambda^{*}>0$ be the number given in Proposition 4.2. For each $\lambda>\lambda^{*}$ and $n \in \mathbb{Z}$, one can find a positive $\delta_{1}>0$ such that for any $T \in \mathcal{R}_{1}(\Omega)$ with $\partial T=0$ in $\Omega$

$$
0<\left\|T-S_{n}\right\|_{0,1}^{*} \leqslant \delta_{1} \Rightarrow \mathcal{G}_{\lambda}(T)>\mathcal{G}_{\lambda}\left(S_{n}\right) \text {. }
$$

Proof. As pointed out just before this last theorem, the action of $T \in \mathcal{R}_{1}(\Omega)$ (that also satisfies $\partial T=0$ relative to $\Omega$ ) on a vector field is really oriented integration over a countable family of Lipschitz curves (each of them having endpoints on $\partial \Omega$ or being a closed loop within $\Omega$ ). We consider first the simplest possible case: that in which $T(B)$ can be expressed as

$$
T(B)=\int_{\gamma} B \cdot \tau
$$

for a single $\gamma:] 0, M\left[\rightarrow B_{R}^{2,+} \subset \mathbb{R}^{2}\right.$ that does not self intersect, with either both endpoints equal or both on $\partial \Omega$, and $n=1$. It follows easily in this case from Proposition 4.2 that $\mathcal{G}_{\lambda}(T)>\mathcal{G}_{\lambda}\left(S_{1}\right)$ for $\lambda>\lambda^{*}$, unless $T=S_{1}$. This more or less implies that $S_{1}$ is an isolated local minimizer of $\mathcal{G}_{\lambda}$. To show that $S_{n}$, for any integer $n \geqslant 1$, actually has this property with respect to $\|\cdot\|_{0,1}^{*}$ we first reduce the problem to the half plane $\left\{\left(x_{1}, x_{2}, x_{3}\right): x_{1} \geqslant 0, x_{2}=0\right\}$, and then use the condition $0<\left\|T-S_{n}\right\|_{0,1}^{*} \leqslant \delta_{1}$ to decompose $T=T_{1}+T_{2}$ where $T_{1}$ is made up of exactly $n$ Lipschitz curves like the ones considered above and $\mathcal{G}_{\lambda}\left(T_{2}\right) \geqslant 0$ with strict inequality if $T_{2} \neq 0$. This will give $\mathcal{G}_{\lambda}(T)>\mathcal{G}_{\lambda}\left(S_{n}\right)$ for general $T \in \mathcal{R}_{1}(\Omega)$ with $\partial T=0$ and $0<\left\|T-S_{n}\right\|_{0,1}^{*} \leqslant \delta_{1}$.

Step 1. Case of single curve. We assume here that $T$ can be represented as

$$
T(B)=T_{\gamma}(B)=\int_{\gamma} B \cdot \tau
$$


for a single curve

$$
\gamma:] 0, M\left[\rightarrow B_{R}^{2,+}=\left\{\left(x_{1}, x_{3}\right) \in \mathbb{R}^{2}: x_{1}^{2}+x_{3}^{2}<R^{2}, x_{1} \geqslant 0\right\}\right.
$$

that does not self intersect and has either both endpoints on $\partial B_{R}^{2}(0)$ or is a closed loop. In this case we may easily associate $T$ with a Cacciopoli set $A_{T} \subset B_{R}^{2,+}$, as in Section 4.1, whose boundary within $B_{R}^{2,+}$ coincides with $\gamma$, and so that also

$$
\mathcal{G}_{\lambda}(T)=\widetilde{\mathcal{G}_{\lambda}}\left(\chi_{A_{T}}\right) \text {. }
$$

In this case Proposition 4.2 immediately gives

$$
\mathcal{G}_{\lambda}(T)>\mathcal{G}_{\lambda}\left(S_{1}\right)
$$

unless $T=S_{1}\left(\right.$ recall $\left.\lambda>\lambda^{*}\right)$.

Step 2 . In this step we show that the energy $\mathcal{G}_{\lambda}$ of any current decreases if we project it along the azimuthal angle onto $B_{R}^{2,+} \subset \mathbb{R}^{2}$. This reduces the problem to a 2 dimensional situation.

This projection, that can also be found for instance in [12], can be described as follows: consider a Cartesian system with $\hat{e}_{3}$ in the direction of $h_{\text {ap }}$ so that $h_{\text {ap }}=\lambda_{\text {ap }} \hat{e}_{3}$. With this, set up a spherical system so that its polar axis coincides with the positive $x_{3}$ axis. We denote these coordinates and unit vectors $(r, \theta, \phi)$ and $\hat{e}_{r}, \hat{e}_{\theta}$ and $\hat{e}_{\phi}$ respectively. In this case $\theta \in[0, \pi]$ is the polar angle. Consider now the map

$$
q: B_{R}(0) \subset \mathbb{R}^{3} \rightarrow B_{R}^{2}=\left\{\left(x_{1}, x_{3}\right): x_{1}^{2}+x_{3}^{2} \leqslant R^{2}\right\} \subset \mathbb{R}^{2}
$$

defined for $x=(r, \theta, \phi) \in B_{R}(0)$ as $q(r, \theta, \phi)=(r \sin \theta, r \cos \theta)$. Looking at the domain of $\theta$ we see that $q\left(B_{R}(0)\right) \subset B_{R}^{2,+}$.

Note now that any vector field $B \in C_{T}^{0,1}\left(B_{R}^{2} ; \mathbb{R}^{2}\right)$ can be readily made into a vector field in $C_{T}^{0,1}\left(B_{R}(0) ; \mathbb{R}^{3}\right)$ (here $B_{R}(0) \subset \mathbb{R}^{3}$ ) independent of the azimuthal coordinate $\phi$, and this is obviously a subset of $C_{T}^{0,1}\left(B_{R}(0)\right.$; $\left.\mathbb{R}^{3}\right)$. This yields

$$
\left\|T_{\gamma}-S_{1}\right\|_{0,1}^{*} \leqslant\left\|T_{\gamma}-S_{1}\right\|_{0,1}^{*} \leqslant \delta_{1},
$$

where the first $\|\cdot\|_{0,1}^{*}$ is understood in $\left(C^{0,1}\left(B_{R}^{2} ; \mathbb{R}^{2}\right)\right)^{*}$ and the second in $\left(C^{0,1}\left(B_{R}(0) ; \mathbb{R}^{3}\right)\right)^{*}$. It is also an easy matter to check $\mathcal{G}_{\lambda}(T) \geqslant \mathcal{G}_{\lambda}\left(q_{\#}(T)\right)$ and $\partial q_{\#}(T)=q_{\#}(\partial T)=0$ relative to $B_{R}^{2}$. All this shows that, to establish the theorem, it suffices to consider $T \in \mathcal{R}_{1}\left(B_{R}^{2}\right)$ with $\operatorname{supp}(T) \subset B_{R}^{2,+}, 0<\left\|T_{\gamma}-S_{1}\right\|_{0,1}^{*} \leqslant \delta_{1}$ and $\partial T=0$ all relative to $B_{R}^{2}$, which we do from now on (see (4.10) and the comment preceding it for the definition of the support of $T$ ).

Step 3. In the next step we decompose $T=T_{1}+T_{2}$, where $T_{1}$ is made up of Lipschitz curves as those considered in Step 1, and $T_{2} \in \mathcal{R}_{1}(\Omega)$ is supported, roughly speaking, on closed loops. To obtain this decomposition we require a lower bound on the mass of $T$, which is what we pursue in this step. More specifically, we seek here the lower bound

$$
M(T) \geqslant M\left(T\left\llcorner\left\{\Gamma^{+} \cap \operatorname{supp}(\psi)\right\}\right) \geqslant 2 n R-C \delta_{1}^{1 / 4} .\right.
$$

for the mass $M(T)$ of any current $T$ that satisfies $\partial T=0$ in $\Omega, 0<\left\|T-S_{n}\right\|_{0,1}^{*} \leqslant \delta_{1}$, and an additional condition that will be clear in a few lines. Here $T\left\llcorner B\right.$ refers to the action of $T$ restricted to the set $B$, the set $\Gamma^{+}$represents a place where $T$ has an orientation close to $S_{n}$, and $\psi$ is a particular test vector field. Both will be defined in the course of this step.

Note first that for $T$ satisfying $0<\left\|T-S_{n}\right\|_{0,1}^{*} \leqslant \delta_{1}$ we have

$$
\left|\left(S_{n}-T\right)\left(B^{*}\right)\right| \leqslant\left\|T-S_{n}\right\|_{0,1}^{*}\left\|B^{*}\right\|_{C^{0,1}} \leqslant \delta_{1}\left\|B^{*}\right\|_{C^{0,1}} .
$$

$M(T) \geqslant M\left(S_{n}\right)+\delta_{1}^{1 / 4}$ implies then that

$$
\begin{aligned}
\mathcal{G}_{\lambda}(T) & =M(T)-2 \lambda T\left(B^{*}\right)=M(T)+2 \lambda\left(S_{n}-T\right)\left(B^{*}\right)-2 \lambda S_{n}\left(B^{*}\right) \\
& \geqslant M\left(S_{n}\right)-2 \lambda S_{n}\left(B^{*}\right)+\delta_{1}^{1 / 4}-2 \lambda \delta_{1}\left\|B^{*}\right\|_{C^{0,1}}>\mathcal{G}_{\lambda}\left(S_{n}\right)
\end{aligned}
$$

for $\delta_{1}=\delta_{1}\left(B^{*}, \lambda\right)>0$ small enough. We therefore assume, in addition to $\partial T=0$ in $\Omega$ and $0<\left\|T-S_{n}\right\|_{0,1}^{*} \leqslant \delta_{1}$, the upper bound

$$
M(T) \leqslant M\left(S_{n}\right)+\delta_{1}^{1 / 4}
$$


throughout the rest of the proof.

To obtain a lower bound on $M(T)$ we build a test vector field with some ideas borrowed from [19]. Let $\alpha>0$ and consider the functions

$$
\begin{aligned}
& f(z)= \begin{cases}R+z & \text { for } z \in]-R,-R+\delta_{1}^{\alpha}[, \\
\delta_{1}^{\alpha} & \text { for } z \in]-R+\delta_{1}^{\alpha}, R-\delta_{1}^{\alpha}[, \\
R-z & \text { for } z \in] R-\delta_{1}^{\alpha}, R[,\end{cases} \\
& \rho(r)=\left\{\begin{array}{ll}
\delta_{1}^{\alpha} & \text { for } r \in] 0, R-\delta_{1}^{\alpha}[, \\
R-r & \text { for } r \in] R-\delta_{1}^{\alpha}, R[,
\end{array}\right. \text { and } \\
& h(x)= \begin{cases}\delta_{1}^{\alpha}-|x| & \text { for }|x| \in\left[0, \delta_{1}^{\alpha}[\right. \\
0 & \text { otherwise. }\end{cases}
\end{aligned}
$$

Here $r=\left(x_{1}^{2}+x_{3}^{2}\right)^{1 / 2}$. Define with these $\psi\left(x_{1}, x_{3}\right)=h\left(x_{1}\right) \rho(r) f\left(x_{3}\right) \hat{e}_{3}$. We compute

$$
S_{n}(\psi)=n \delta_{1}^{3 \alpha}\left(2 R-2 \delta_{1}^{\alpha}\right)+\frac{2 n \delta_{1}^{4 \alpha}}{3}=n \delta_{1}^{3 \alpha}\left(2 R-\frac{4}{3} \delta_{1}^{\alpha}\right) .
$$

It is also clear that $\|\psi\|_{C^{0,1}} \leqslant 1$. Since $\left|\left(S_{n}-T\right)(\psi)\right| \leqslant \delta_{1}\|\psi\|_{C^{0,1}}$, this implies that

$$
T(\psi) \geqslant S_{n}(\psi)-\delta_{1}=n \delta_{1}^{3 \alpha}\left(2 R-\frac{4}{3} \delta_{1}^{\alpha}\right)-\delta_{1} .
$$

Let us introduce the following notation.

$$
\Gamma^{+}=\left\{x \in \Gamma: \hat{e}_{3} \cdot \hat{\tau}_{x}>0\right\} .
$$

The definitions of $\psi$ and $\Gamma^{+}$lead to

$$
T(\psi) \leqslant \delta_{1}^{3 \alpha} M\left(T\left\llcorner\left\{\Gamma^{+} \cap \operatorname{supp}(\psi)\right\}\right)\right.
$$

and from here we obtain that

$$
M\left(T\left\llcorner\left\{\Gamma^{+} \cap \operatorname{supp}(\psi)\right\}\right) \geqslant\left(2 n R-\frac{4 n}{3} \delta_{1}^{\alpha}\right)-\delta_{1}^{1-3 \alpha} \geqslant 2 n R-C \delta_{1}^{\min \{\alpha, 1-3 \alpha\}}\right.
$$

for $\delta_{1}=\delta_{1}(R, n)>0$ small enough. Choosing now $\alpha=\frac{1}{4}$, we obtain

$$
M\left(T\left\llcorner\left\{\Gamma^{+} \cap \operatorname{supp}(\psi)\right\}\right) \geqslant 2 n R-C \delta_{1}^{1 / 4},\right.
$$

which is (4.11).

Step 4. As mentioned before, here we use Step 3 to decompose $T=T_{1}+T_{2}$, where $T_{1}$ is a current supported on finitely many single Lipschitz curves, and $T_{2} \in \mathcal{R}_{1}(\Omega)$ is basically supported on closed loops. To do this we first recall from 4.2.25 in [7] that $T \in \mathcal{R}_{1}(\Omega)$ with $\partial T=0$ can be decomposed as

$$
T=\sum_{k \geqslant 1} T_{k}
$$

Furthermore, each $T_{k}$ is a single Lipschitz curve with both endpoints on $\partial \Omega$ or else is a closed curve within $\Omega$ (either way $\partial T_{k}=0$ for all $k$ ), and one also has $M(T)=\sum_{k \geqslant 1} M\left(T_{k}\right)$. We also recall from [7] that for $f: \Omega \rightarrow \mathbb{R}$ Lipschitz, the slices $\langle T, f, t\rangle$ are well defined for a.e $t \in \mathbb{R}$. Loosely speaking, for $T \in \mathcal{R}_{1}(\Omega),\langle T, f, t\rangle$ represents the restriction of $T$ to the surface $f^{-1}(t) \subset \Omega$. Since $T \in \mathcal{R}_{1}(\Omega)$ is made up of a countable collection of Lipschitz curves, for a.e. $t \in \mathbb{R},\langle T, f, t\rangle$ is a countable collection of point masses and

$$
\int_{-\infty}^{\infty} M(\langle T, f, t\rangle) \mathrm{d} t \leqslant \sup _{x \in \Gamma}\left|\nabla^{\Gamma} f(x)\right| M(T) .
$$

Here we recall that the set $\Gamma=\operatorname{support}(T)$ and $\nabla^{\Gamma} f(x)$ represents the component of $\nabla f$ tangent to $\Gamma$. 
Now we try to compare the support of $T$ with that of $S_{n}$. Set $g\left(x_{1}, x_{3}\right)=\left|x_{1}\right|$ and let $C^{\prime}>C$ where $C>0$ comes from (4.11) (and depends only on the multiplicity $n$ of $S_{n}$ ). Let us first rule out the possibility that for a.e. $r \in\left[\delta_{1}^{\alpha}, 2 C^{\prime} \delta_{1}^{\alpha}\right]$ it holds

$$
M(\langle T, g, r\rangle) \geqslant 1 \text {. }
$$

Indeed, since $\operatorname{supp}(\psi) \cap g^{-1}\left(\left[\delta_{1}^{\alpha}, 2 C^{\prime} \delta_{1}^{\alpha}\right]\right)=\emptyset$ (recall $\left.\alpha=1 / 4\right)$, we find, in light of (4.11) and (4.14), that (4.15) leads to

$$
M(T) \geqslant M\left(T\left\llcorner\left\{\Gamma^{+} \cap \operatorname{supp}(\psi)\right\}\right)+M\left(T \left\llcornerg^{-1}\left(\left[\delta_{1}^{\alpha}, 2 C^{\prime} \delta_{1}^{\alpha}\right]\right) \geqslant 2 n R-C \delta_{1}^{\alpha}+\left(2 C^{\prime}-1\right) \delta_{1}^{\alpha} .\right.\right.\right.
$$

For $C^{\prime}>0$ large enough (again depending only on $n$ ) this contradicts $M(T) \leqslant 2 n R+\delta_{1}^{1 / 4}$ for $\delta_{1}>0$ small enough. It follows that

$$
H^{(1)}\left(\left\{r \in\left[\delta_{1}^{\alpha}, C \delta_{1}^{\alpha}\right]: M(\langle T, g, r\rangle)=0\right\}\right)>0,
$$

where we relabel $2 C^{\prime}$ to $C$. Define the set $\sigma=\left\{k \in \mathbb{N}: T_{k}(\psi) \neq 0\right\}$. (4.16) ensures that for $k \in \sigma, T_{k}$ is a curve contained in the interior of the infinite cylinder $g^{-1}\left(\left[0, C \delta_{1}^{\alpha}[)\right.\right.$. We subdivide $\sigma$ further by considering

$$
\begin{aligned}
\sigma_{2}= & \left\{k \in \sigma: T_{k} \text { has both ends on the same connected component of } \partial B_{R}^{2} \cap g^{-1}(] 0, C \delta_{1}^{\alpha}[)\right. \text { or } \\
& \left.T_{k} \text { is a closed loop }\right\}
\end{aligned}
$$

and $\sigma_{1}=\sigma \backslash \sigma_{2}$. Note that for $k \in \sigma_{1}, T_{k}$ is a curve with one endpoint on each connected component of $\partial B_{R}^{2} \cap$ $g^{-1}(] 0, C \delta_{1}^{\alpha}[)$, and hence

$$
M\left(T_{k}\right) \geqslant 2 R-C \delta_{1}^{\alpha}
$$

for each $k \in \sigma_{1}$. The assumption that $M(T) \leqslant 2 n R+\delta_{1}^{1 / 4}$ guarantees then that there are at most $n$ integers in $\sigma_{1}$. We distinguish two cases: $\operatorname{card}\left(\sigma_{1}\right)=n$ and $\operatorname{card}\left(\sigma_{1}\right)<n$.

Step 5. Impossibility of $m=\operatorname{card}\left(\sigma_{1}\right)<n$. We follow the proof of Theorem 4.5 from [19]. In this case one can conclude that in fact $M(T) \geqslant 2 n R+(n-m) R-C \delta_{1}^{\gamma}$ for some $\gamma>0$. This clearly contradicts $M(T) \leqslant 2 n R+C \delta_{1}^{1 / 4}$ for $\delta_{1}=\delta_{1}(n, m, R)>0$ small enough. The details of the proof are as Theorem 4.5 from [19] so we omit them here. Roughly speaking though, the main idea can be expressed as follows: because there are only $m<n$ integers in $\sigma_{1}$, (4.11) implies that there must be $2(n-m) R$ units of mass in $T$ that come from the portion of either closed loops or curves that have both end-points on the same connected component of $\partial \Omega \cap g^{-1}(] 0,2 \delta_{1}^{\beta}[)$ that lies in $\Gamma^{+}$. These curves however will have at least as much mass in the portion of them that lies in $\Gamma^{-}=\left\{x \in \operatorname{supp}(T):-\hat{\tau}_{x} \cdot \hat{e}_{3} \geqslant 0\right\}$, this is at least $2(n-m) R$ units of mass in $\Gamma^{-}$. These are unaccounted for in (4.11). Careful book-keeping then leads to $M(T) \geqslant 2 n R+(n-m) R-C \delta_{1}^{\gamma}$ for some $\gamma>0$ which, as mentioned earlier, is impossible.

Step 6. Conclusion. In light of Step 5 we assume $\operatorname{card}\left(\sigma_{1}\right)=n$. Note also that for $k \in \sigma_{1}$ Step 1 implies that $\mathcal{G}_{\lambda}\left(T_{k}\right)-\mathcal{G}_{\lambda}\left(S_{1}\right)>0$ unless $T_{k}=S_{1}$.

To estimate $\mathcal{G}_{\lambda}\left(T-T_{\sigma_{1}}\right)$ we note that

$$
M\left(T_{\sigma_{1}}\right)=\sum_{k \in \sigma_{1}} M\left(T_{k}\right) \geqslant 2 n R-C \delta_{1}^{\alpha} .
$$

This implies that $M\left(T-T_{\sigma_{1}}\right)=\sum_{\mathbb{N} \backslash \sigma_{1}} M\left(T_{k}\right) \leqslant C \delta_{1}^{\alpha}$, in light of $M(T) \leqslant 2 n R+C \delta_{1}^{1 / 4}$. We can find then an integer multiplicity 2-current $S$ with $\partial S=T-T_{\sigma_{1}}$. The relative isoperimetric inequality gives in this case that $M(S) \leqslant$ $K\left(M\left(T-T_{\sigma_{1}}\right)\right)^{2}$. But then

$$
\mathcal{G}_{\lambda}\left(T-T_{\sigma_{1}}\right)=M\left(T-T_{\sigma_{1}}\right)-2 \lambda \int_{S} n_{S}(x)\left(\nabla \times B^{*}\right) \cdot v \mathrm{~d} S \geqslant M\left(T-T_{\sigma_{1}}\right)-K \lambda\left\|\nabla \times B^{*}\right\|_{\infty}\left(M\left(T-T_{\sigma_{1}}\right)\right)^{2}
$$

and since $M\left(T-T_{\sigma_{1}}\right)=\sum_{\mathbb{N} \backslash \sigma_{1}} M\left(T_{k}\right) \leqslant C \delta_{1}^{\alpha}$, this clearly implies that $\mathcal{G}_{\lambda}\left(T-T_{\sigma_{1}}\right)>0$, unless $T-T_{\sigma_{1}}=0$. We can conclude now since (recall card $\left.\left(\sigma_{1}\right)=n\right)$

$$
\mathcal{G}_{\lambda}(T)-\mathcal{G}_{\lambda}\left(S_{n}\right)=\sum_{k \in \sigma_{1}}\left(\mathcal{G}_{\lambda}\left(T_{k}\right)-\mathcal{G}_{\lambda}\left(S_{1}\right)\right)+\mathcal{G}_{\lambda}\left(T-T_{\sigma_{1}}\right)
$$


and either $T-T_{\sigma_{1}}=0$, in which case $T \neq S_{n}$ implies by step 1 that $\mathcal{G}_{\lambda}\left(T_{k_{0}}\right)-\mathcal{G}_{\lambda}\left(S_{1}\right)>0$ for some $k_{0} \in \sigma_{1}$ (recall $\mathcal{G}_{\lambda}\left(T_{k}\right)-\mathcal{G}_{\lambda}\left(S_{1}\right) \geqslant 0$ for all $k \in \sigma_{1}$ with strict inequality if $T_{k} \neq S_{1}$ by Step 1$)$, or $T-T_{\sigma_{1}} \neq 0$, in which case $\mathcal{G}_{\lambda}\left(T-T_{\sigma_{1}}\right)>0$. In both instances we obtain $\mathcal{G}_{\lambda}(T)-\mathcal{G}_{\lambda}\left(S_{n}\right)>0$, which is the claim of the theorem.

\section{Local minimizers to the Ginzburg-Landau energy}

In this section we prove Theorem 1.1, by building local minimizers to (1.1). We note as in [13] that a sort of singular change of gauge on $G_{\varepsilon}(u, A)$ leads to an expression for it that can be handled using weak Jacobians. We recall here that the gauge invariance of $G_{\varepsilon}$ is a property that reads

$$
G_{\varepsilon}\left(\mathrm{e}^{\mathrm{i} \phi} u, A+\nabla \phi\right)=G_{\varepsilon}(u, A)
$$

whenever $\phi \in W^{1,2}\left(\mathbb{R}^{3}\right)$. Instead of computing the left-hand side of this last identity we compute

$$
\mathcal{G}_{\varepsilon}(u, A)=G_{\varepsilon}\left(\mathrm{e}^{\mathrm{i} \phi_{A}} u, A\right),
$$

for $\phi_{A}$ coming from

$$
A=\nabla \times B_{A}+\nabla \phi_{A}
$$

(cf. Lemma 2.4). Note that this still makes sense, although $\phi_{A}$ is not defined in all of $\mathbb{R}^{3}$. An easy computation yields

$$
\mathcal{G}_{\varepsilon}(u, A)=E_{\varepsilon}(u)-2 \int_{\Omega} B_{A} \cdot J(u) \mathrm{d} x+\frac{1}{2} \int_{\Omega}|u|^{2}\left|\nabla \times B_{A}\right|^{2} \mathrm{~d} x+\frac{1}{2} \int_{\mathbb{R}^{3}}\left|\nabla \times A-h_{\mathrm{ap}}^{\varepsilon}\right|^{2} \mathrm{~d} x,
$$

where

$$
E_{\varepsilon}(u)=\frac{1}{2} \int_{\Omega}|\nabla u|^{2} \mathrm{~d} x+\frac{1}{4 \varepsilon^{2}} \int_{\Omega}\left(1-|u|^{2}\right)^{2} \mathrm{~d} x .
$$

This $\mathcal{G}_{\varepsilon}(u, A)$ of course is not the Ginzburg-Landau energy. However the transformation

$$
\begin{aligned}
\mathcal{T}: W^{1,2}(\Omega ; \mathbb{C}) \times H_{0} & \rightarrow W^{1,2}(\Omega ; \mathbb{C}) \times H_{0}, \\
(u, A) & \rightarrow \mathcal{T}(u, A)=\left(\mathrm{e}^{\mathrm{i} \phi_{A}} u, A\right)
\end{aligned}
$$

is a diffeomorphism (cf. [13]). This means that local minimizers to $G_{\varepsilon}(u, A)$ defined by (1.1) produce local minimizers to $\mathcal{G}_{\varepsilon}(u, A)$ and vice versa. This allows us to study $\mathcal{G}_{\varepsilon}$, which is what the next theorem talks about.

In the following, we take $h_{\mathrm{ap}}^{\varepsilon}=\lambda|\ln \varepsilon| \hat{e}_{3}$, and define $A_{\mathrm{ap}}^{\varepsilon}$ with $\nabla \times A_{\mathrm{ap}}^{\varepsilon}=h_{\mathrm{ap}}^{\varepsilon}$ and $B_{\mathrm{ap}}^{\varepsilon}$ as in Lemma 2.4. We decompose our magnetic potentials $A=A_{\text {ap }}^{\varepsilon}+A_{1}$ with $A_{1} \in H_{0}$. Denote by $\mathcal{R}_{1}(\Omega)$ the class of integer multiplicity rectifiable one currents. $S_{n} \in \mathcal{R}_{1}\left(B_{R}(0)\right)$ denotes the current defined as the vertical diameter $\left\{\left(0,0, x_{3}\right):-R<x_{3}<\right.$ $R\}$ of the ball $\Omega=B_{R}(0)$, with integer multiplicity $n$.

We recall from the Introduction the following notation: for $\delta>0$ define,

$$
\begin{aligned}
& \mathcal{F}=\mathcal{F}_{\delta}=\left\{\left(u, A_{1}\right) \in W^{1,2}(\Omega ; \mathbb{C}) \times H_{0}:\left\|S_{n}-\frac{1}{\pi} J(u)\right\|_{1, p}^{*} \leqslant \delta\right\}, \\
& \mathcal{O}=\mathcal{O}_{\delta}=\left\{\left(u, A_{1}\right) \in W^{1,2}(\Omega ; \mathbb{C}) \times H_{0}:\left\|S_{n}-\frac{1}{\pi} J(u)\right\|_{1, p}^{*}<\delta\right\} .
\end{aligned}
$$

We claim that $\mathcal{F}$ is weakly closed and $\mathcal{O}$ is open in $W^{1,2}(\Omega ; \mathbb{C}) \times H_{0}$. The proof of these two facts follows that of Theorem 4.2 from [19], with the only caveat that the proofs in [19] are for $\|\cdot\|_{0,1}^{*}$. The difference is minor so we do not include the proof here.

Note first that, for $B \in C^{\infty}\left(\Omega ; \mathbb{R}^{3}\right)$ and $p>3$ one has

$$
\|B\|_{C^{0, \alpha}} \leqslant K_{1}\|B\|_{W^{1, p}} \leqslant K_{2}\|B\|_{C^{0,1}}
$$

where $\alpha=1-\frac{3}{p}$. We set here $p=4$ and $\alpha=\frac{1}{4}$. This implies for $T \in\left(C_{T}^{0, \alpha}\left(\Omega ; \mathbb{R}^{3}\right)\right)^{*}$ that

$$
\|T\|_{0,1}^{*} \leqslant K_{2}\|T\|_{1, p}^{*} \leqslant K_{1} K_{2}\|T\|_{0, \alpha}^{*} .
$$


Here $\|T\|_{0, \alpha}^{*}$ and $\|T\|_{1, p}^{*}$ represent the norms on $T$ dual to the usual Hölder norm $\|\cdot\|_{C^{0, \alpha}}$ and dual to the Sobolev norm $\|\cdot\|_{W^{1, p}}$ respectively.

Next we apply the direct method of the calculus of variations to the problem of finding $\left(u_{\varepsilon}, A_{1, \varepsilon}\right) \in \mathcal{F}$ satisfying

$$
\mathcal{G}_{\varepsilon}\left(u_{\varepsilon}, A_{1, \varepsilon}+A_{\text {ap }}^{\varepsilon}\right)=\inf _{\left(u, A_{1}\right) \in \mathcal{F}} \mathcal{G}_{\varepsilon}\left(u, A_{1}+A_{\text {ap }}^{\varepsilon}\right) .
$$

Since $\mathcal{F}$ is weakly closed, it is a simple matter to obtain the existence of a solution to this last problem. The remainder of the proof consists in showing that in fact $\left(u_{\varepsilon}, A_{1, \varepsilon}\right) \in \mathcal{O}$ for $\varepsilon>0$ small enough. We proceed by contradiction and assume that there is a sequence $\varepsilon_{n} \rightarrow 0$ with $\left\|S_{n}-\frac{1}{\pi} J\left(u_{\varepsilon_{n}}\right)\right\|_{1,4}^{*}=\delta_{0}$. From now on we drop the subscript $n$ and write $\left(u_{\varepsilon}, A_{1, \varepsilon}\right)$ for $\left(u_{\varepsilon_{n}}, A_{1, \varepsilon_{n}}\right)$. We will take several steps.

Step 1. From [2] and [13], one can always find a sequence $\left\{v_{\varepsilon}\right\} \subset W^{1,2}(\Omega ; \mathbb{C})$ with $E_{\varepsilon}\left(v_{\varepsilon}\right) \leqslant K \ln \frac{1}{\varepsilon}$, and

$$
\begin{aligned}
& \lim _{\varepsilon \rightarrow 0} E_{\varepsilon}\left(v_{\varepsilon}\right)=M\left(S_{n}\right), \\
& \lim _{\varepsilon \rightarrow 0} \frac{1}{\pi} J\left(v_{\varepsilon}\right)=S_{n}
\end{aligned}
$$

and the last convergence is strong in $\left(C_{T}^{0, \beta}\left(\Omega ; \mathbb{R}^{3}\right)\right)^{*}$, for any $\left.\left.\beta \in\right] 0,1\right]$. In particular, from (5.5), this convergence also is strong in $\left(W_{T}^{1,4}\left(\Omega ; \mathbb{R}^{3}\right)\right)^{*}$. Clearly then $\left(v_{\varepsilon}, 0\right) \in \mathcal{F}$ for $\varepsilon>0$ small enough. This implies that $\mathcal{G}_{\varepsilon}\left(u_{\varepsilon}, A_{1, \varepsilon}+A_{\text {ap }}^{\varepsilon}\right) \leqslant$ $\mathcal{G}_{\varepsilon}\left(v_{\varepsilon}, 0+A_{\text {ap }}^{\varepsilon}\right) \leqslant K\left(\ln \frac{1}{\varepsilon}\right)^{2}$, and this in turn yields

$$
\int_{\mathbb{R}^{3}}\left|\nabla \times A_{1, \varepsilon}\right|^{2} \mathrm{~d} x \leqslant K\left(\ln \frac{1}{\varepsilon}\right)^{2},
$$

and

$$
E_{\varepsilon}\left(u_{\varepsilon}\right) \leqslant K\left(\ln \frac{1}{\varepsilon}\right)^{2} .
$$

Now writing $B_{1, \varepsilon}=B_{A_{1, \varepsilon}}$, we obtain from Lemma 2.4 that

$$
\left\|B_{1, \varepsilon}\right\|_{W^{2,2}}^{2} \leqslant K\left(\ln \frac{1}{\varepsilon}\right)^{2} \text {. }
$$

Step 2. Clearly $\left(v_{\varepsilon}, A_{1, \varepsilon}\right) \in \mathcal{F}$ also, so $\mathcal{G}_{\varepsilon}\left(u_{\varepsilon}, A_{1, \varepsilon}+A_{\text {ap }}^{\varepsilon}\right) \leqslant \mathcal{G}_{\varepsilon}\left(v_{\varepsilon}, A_{1, \varepsilon}+A_{\text {ap }}^{\varepsilon}\right)$. After some cancellation one concludes from here

$$
\begin{aligned}
E_{\varepsilon}\left(u_{\varepsilon}\right) \leqslant & E_{\varepsilon}\left(v_{\varepsilon}\right)-2\left(J\left(v_{\varepsilon}\right)-J\left(u_{\varepsilon}\right)\right)\left(B_{1, \varepsilon}+B_{\mathrm{ap}}^{\varepsilon}\right)+\int_{\Omega}\left(1-\left|u_{\varepsilon}\right|^{2}\right)\left|\nabla \times\left(B_{1, \varepsilon}+B_{\mathrm{ap}}^{\varepsilon}\right)\right|^{2} \mathrm{~d} x \\
& \quad-\int_{\Omega}\left(1-\left|v_{\varepsilon}\right|^{2}\right)\left|\nabla \times\left(B_{1, \varepsilon}+B_{\mathrm{ap}}^{\varepsilon}\right)\right|^{2} \mathrm{~d} x \\
\leqslant & E_{\varepsilon}\left(v_{\varepsilon}\right)+2\left\|J\left(v_{\varepsilon}\right)-J\left(u_{\varepsilon}\right)\right\|_{1, p}^{*}\left\|B_{1, \varepsilon}+B_{\mathrm{ap}}^{\varepsilon}\right\|_{W^{1, p}}+\int_{\Omega}\left(1-\left|u_{\varepsilon}\right|^{2}\right)\left|\nabla \times\left(B_{1, \varepsilon}+B_{\mathrm{ap}}^{\varepsilon}\right)\right|^{2} \mathrm{~d} x \\
& -\int_{\Omega}\left(1-\left|v_{\varepsilon}\right|^{2}\right)\left|\nabla \times\left(B_{1, \varepsilon}+B_{\mathrm{ap}}^{\varepsilon}\right)\right|^{2} \mathrm{~d} x .
\end{aligned}
$$

Here we point out that

$$
\begin{aligned}
\int_{\Omega}\left(1-\left|u_{\varepsilon}\right|^{2}\right)\left|\nabla \times\left(B_{1, \varepsilon}+B_{\mathrm{ap}}^{\varepsilon}\right)\right|^{2} \mathrm{~d} x & \leqslant\left(\int_{\Omega}\left(1-\left|u_{\varepsilon}\right|^{2}\right)^{2} \mathrm{~d} x \int_{\Omega}\left(\left|\nabla \times\left(B_{1, \varepsilon}+B_{\mathrm{ap}}^{\varepsilon}\right)\right|^{4} \mathrm{~d} x\right)^{1 / 2}\right. \\
& \leqslant C \varepsilon\left(E_{\varepsilon}\left(u_{\varepsilon}\right)\right)^{1 / 2}\left\|B_{1, \varepsilon}+B_{\mathrm{ap}}^{\varepsilon}\right\|_{W^{2,2}}^{2} \leqslant C \varepsilon\left(\ln \frac{1}{\varepsilon}\right)^{3},
\end{aligned}
$$


where we used (5.10), (5.9) and $B_{\text {ap }}^{\varepsilon}=|\ln \varepsilon| B_{\text {ap }}$. Similarly we obtain

$$
\int_{\Omega}\left(1-\left|v_{\varepsilon}\right|^{2}\right)\left|\nabla \times\left(B_{1, \varepsilon}+B_{\mathrm{ap}}^{\varepsilon}\right)\right|^{2} \mathrm{~d} x \leqslant C \varepsilon\left(\ln \frac{1}{\varepsilon}\right)^{3} .
$$

Also note that since $p=4$, we have

$$
\left\|B_{1, \varepsilon}\right\|_{W^{1, p}} \leqslant C\left\|B_{1, \varepsilon}\right\|_{W^{2,2}} \leqslant C|\ln \varepsilon|,
$$

and

$$
\left\|J\left(v_{\varepsilon}\right)-J\left(u_{\varepsilon}\right)\right\|_{1, p}^{*} \leqslant\left\|J\left(v_{\varepsilon}\right)-S_{n}\right\|_{1, p}^{*}+\left\|S_{n}-J\left(u_{\varepsilon}\right)\right\|_{1, p}^{*} \leqslant C .
$$

All of this in (5.12) yields

$$
E_{\varepsilon}\left(u_{\varepsilon}\right) \leqslant K \ln \frac{1}{\varepsilon}
$$

We appeal now to [14] and [13] to claim the existence of a subsequence of $\left\{J\left(u_{\varepsilon}\right)\right\}$ strongly convergent in $\left(C_{T}^{0, \beta}\left(\Omega ; \mathbb{R}^{3}\right)\right)^{*}$ for all $\left.\left.\beta \in\right] 0,1\right]$. (5.5) implies then that this convergence is also strong in $\left(W_{T}^{1,4}\left(\Omega ; \mathbb{R}^{3}\right)\right)^{*}$. Call the limit $\pi T$. From [14] we also conclude that $T$ is an integer multiplicity, rectifiable 1-current. Furthermore, the fact that the convergence is strong in $\left(W_{T}^{1,4}\right)^{*}$ implies that $\left\|S_{n}-T\right\|_{1,4}^{*}=\delta_{0}$, so in particular $T \neq S_{n}$. Finally, [14] also provides the inequality

$$
M(\pi T) \leqslant \liminf _{\varepsilon \rightarrow 0} \frac{1}{|\ln \varepsilon|} E_{\varepsilon}\left(u_{\varepsilon}\right) .
$$

Step 3. By Step 1, $\left\|B_{1, \varepsilon}\right\|_{W^{2,2}}^{2} \leqslant K\left(\ln \frac{1}{\varepsilon}\right)^{2}$. As mentioned earlier, the Sobolev embeddings then imply that $\left\|B_{1, \varepsilon}\right\|_{W^{1,4}} \leqslant K \ln \frac{1}{\varepsilon}$. Moreover, for the exponents we are using the embedding is compact. It follows that $\frac{1}{|\ln \varepsilon|} B_{1, \varepsilon}$ is pre-compact in $W_{T}^{1,4}\left(\Omega ; \mathbb{R}^{3}\right)$. We work now towards identifying the limit of $\frac{1}{|\ln \varepsilon|} B_{1, \varepsilon}$. To this end recall from [13] the

Proposition 5.1. Let $\Omega \subset \mathbb{R}^{3}$ be a smooth domain, and let $\alpha \in(0,1]$. Then there are constants $\gamma>0$ and $C(\alpha, \Omega)>0$ such that for any $v \in W^{1,2}(\Omega ; \mathbb{C})$ and any $\varepsilon \in(0,1)$ one has

$$
\|J(v)\|_{C_{T}^{0, \alpha}(\Omega)^{*}} \leqslant C(\alpha, \Omega)\left(\varepsilon^{\gamma}+\frac{E_{\varepsilon}(v)}{|\ln \varepsilon|}\right) .
$$

We next show that $\frac{1}{|\ln \varepsilon|}\left(B_{1, \varepsilon}+B_{\mathrm{ap}}^{\varepsilon}\right) \rightarrow \lambda B^{*}$, where $B^{*}$ is given by (3.6), corresponding to minimization of the functional $F(A)$ in Theorem 3.1, when $h_{\mathrm{ap}}=\hat{e}_{3}$. To do this note that

$$
\mathcal{G}\left(u_{\varepsilon}, A_{1, \varepsilon}+A_{\text {ap }}^{\varepsilon}\right) \leqslant \mathcal{G}\left(u_{\varepsilon}, \ln \left(\frac{1}{\varepsilon}\right) A_{1}+A_{\text {ap }}^{\varepsilon}\right)
$$

for any $A_{1} \in H_{0}$. In fact, $\left(u_{\varepsilon}, A_{1, \varepsilon}\right) \in \mathcal{F}$ implies

$$
\left\|\frac{1}{\pi} J\left(u_{\varepsilon}\right)-S_{n}\right\|_{1, p}^{*} \leqslant \delta_{1} .
$$

This clearly implies $\left(u_{\varepsilon},|\ln \varepsilon| A_{1}\right) \in \mathcal{F}$ for any $A_{1} \in H_{0}$, so we obtain

$$
\mathcal{G}\left(u_{\varepsilon}, A_{1, \varepsilon}+A_{\mathrm{ap}}^{\varepsilon}\right) \leqslant \mathcal{G}\left(u_{\varepsilon},|\ln \varepsilon| A_{1}+A_{\mathrm{ap}}^{\varepsilon}\right) .
$$

Expanding this last inequality we obtain

$$
\begin{aligned}
& E_{\varepsilon}\left(u_{\varepsilon}\right)-2 \int_{\Omega}\left(B_{1, \varepsilon}+B_{\mathrm{ap}}^{\varepsilon}\right) \cdot J\left(u_{\varepsilon}\right)+\frac{1}{2} \int_{\Omega}\left|u_{\varepsilon}\right|^{2}\left|\nabla \times\left(B_{1, \varepsilon}+B_{\mathrm{ap}}^{\varepsilon}\right)\right|^{2}+\frac{1}{2} \int_{\mathbb{R}^{3}}\left|\nabla \times A_{1, \varepsilon}\right|^{2} \\
& \leqslant E_{\varepsilon}\left(u_{\varepsilon}\right)-2 \int_{\Omega}\left(|\ln \varepsilon| B_{1}+B_{\mathrm{ap}}^{\varepsilon}\right) \cdot J\left(u_{\varepsilon}\right)+\frac{|\ln \varepsilon|^{2}}{2} \int_{\Omega}\left|u_{\varepsilon}\right|^{2}\left|\nabla \times\left(B_{1}+B_{\mathrm{ap}}\right)\right|^{2}
\end{aligned}
$$




$$
+\frac{|\ln \varepsilon|^{2}}{2} \int_{\mathbb{R}^{3}}\left|\nabla \times A_{1}\right|^{2}
$$

Note that $\frac{1}{|\ln \varepsilon|} A_{1, \varepsilon}$ is weakly compact in $H_{0}$ by (5.8). Note also that Proposition 5.1 and (5.14) imply

$$
\frac{1}{|\ln \varepsilon|^{2}} \int_{\Omega}\left(B_{1, \varepsilon}+B_{\mathrm{ap}}^{\varepsilon}\right) \cdot J\left(u_{\varepsilon}\right)=0 .
$$

We divide now (5.17) by $|\ln \varepsilon|^{2}$ and let $\varepsilon \rightarrow 0$. Call

$$
\bar{A}_{1}=w-\lim _{\varepsilon \rightarrow 0} \frac{1}{|\ln \varepsilon|} A_{1, \varepsilon}, \quad \bar{B}_{1}:=B_{A_{1}},
$$

and denote

$$
B_{\text {ap }}=\frac{B_{\text {ap }}^{\varepsilon}}{|\ln \varepsilon|}, \quad A_{\text {ap }}=\frac{A_{\text {app }}^{\varepsilon}}{|\ln \varepsilon|} .
$$

The above discussion reduces then (5.17) to

$$
\frac{1}{2} \int_{\Omega}\left|\nabla \times\left(\bar{B}_{1}+B_{\mathrm{ap}}\right)\right|^{2}+\frac{1}{2} \int_{\mathbb{R}^{3}}\left|\nabla \times \bar{A}_{1}\right|^{2} \leqslant \frac{1}{2} \int_{\Omega}\left|\nabla \times\left(B_{1}+B_{\mathrm{ap}}\right)\right|^{2}+\frac{1}{2} \int_{\mathbb{R}^{3}}\left|\nabla \times A_{1}\right|^{2}
$$

for all $A_{1} \in H_{0}$, so $\bar{A}_{1}$ is the unique minimizer of

$$
F\left(A_{1}\right)=\frac{1}{2} \int_{\Omega}\left|\nabla \times\left(B_{1}+B_{\mathrm{ap}}\right)\right|^{2} \mathrm{~d} x+\frac{1}{2} \int_{\mathbb{R}^{3}}\left|\nabla \times A_{1}\right|^{2} \mathrm{~d} x .
$$

Set now $B_{0}=\bar{B}_{1}+B_{\text {ap }}$ and recall from the remark below (3.6) that $B_{0}=\lambda B^{*}$. We rearrange (5.11) as

$$
\begin{gathered}
E_{\varepsilon}\left(u_{\varepsilon}\right)-2 \int_{\Omega}\left(B_{1, \varepsilon}+B_{\mathrm{ap}}^{\varepsilon}\right) \cdot J\left(u_{\varepsilon}\right) \mathrm{d} x \leqslant \\
E_{\varepsilon}\left(v_{\varepsilon}\right)-2 \int_{\Omega} J\left(v_{\varepsilon}\right) \cdot\left(B_{1, \varepsilon}+B_{\mathrm{ap}}^{\varepsilon}\right)+\int_{\Omega}\left(1-\left|u_{\varepsilon}\right|^{2}\right)\left|\nabla \times B_{1, \varepsilon}\right|^{2} \mathrm{~d} x \\
-\int_{\Omega}\left(1-\left|v_{\varepsilon}\right|^{2}\right)\left|\nabla \times B_{1, \varepsilon}\right|^{2} \mathrm{~d} x .
\end{gathered}
$$

We now use the known compactness of $B_{1, \varepsilon}$, the conclusions for $J\left(u_{\varepsilon}\right)$ mentioned at the end of Step 2, (5.18), (5.6) and (5.7) to conclude

$$
\begin{aligned}
M(T)-2 \lambda T\left(B_{*}\right) & \leqslant \liminf _{\varepsilon \rightarrow 0}\left\{\frac{E_{\varepsilon}\left(u_{\varepsilon}\right)}{\pi|\ln \varepsilon|}-\frac{2}{|\ln \varepsilon|} \int_{\Omega}\left(B_{1, \varepsilon}+B_{\text {ap }}^{\varepsilon}\right) \cdot J\left(u_{\varepsilon}\right) \mathrm{d} x\right\} \\
& \leqslant \liminf _{\varepsilon \rightarrow 0}\left\{\frac{E_{\varepsilon}\left(v_{\varepsilon}\right)}{\pi|\ln \varepsilon|}-\frac{2}{|\ln \varepsilon|} \int_{\Omega}\left(B_{1, \varepsilon}+B_{\text {ap }}^{\varepsilon}\right) \cdot J\left(v_{\varepsilon}\right) \mathrm{d} x\right\} \\
& \leqslant M\left(S_{n}\right)-2 \lambda S_{n}\left(B_{*}\right) .
\end{aligned}
$$

However, from Step 2, $S_{n} \neq T$. Also from Step 2, $\left\|S_{n}-T\right\|_{1,4}^{*}=\delta_{0}$, so (5.5) implies that $\left\|S_{n}-T\right\|_{0,1}^{*} \leqslant K_{2} \delta_{0}$. Theorem 4.3 then yields a contradiction for $\delta_{0}>0$ small enough, because $0<\left\|S_{n}-T\right\|_{0,1}^{*} \leqslant K_{2} \delta_{0}$ implies that

$$
M(T)-2 \lambda T\left(B_{*}\right)=\mathcal{G}_{\lambda}(T)>\mathcal{G}_{\lambda}\left(S_{n}\right)=M\left(S_{n}\right)-2 \lambda S_{n}\left(B_{*}\right) .
$$

Step 4 . The only details that still needs a proof are $\frac{1}{\pi} J\left(u_{\varepsilon}\right) \rightarrow S_{n}$ and the fact that for every $\eta>0$ there is $\varepsilon_{0}>0$ such that, for every $0<\varepsilon \leqslant \varepsilon_{0}$,

$$
\operatorname{supp}\left(S_{1}\right) \subset\left\{x \in \Omega: \operatorname{dist}\left(x, N_{1 / 2}^{\varepsilon}\right) \leqslant \eta\right\} .
$$

Here

$$
N_{1 / 2}^{\varepsilon}=\left\{x \in \mathbb{R}^{3}:\left|u_{\varepsilon}(x)\right|<1 / 2\right\} .
$$


For the first one note that the same contradiction we reached in the course of steps 1 through 3 would have been reached if we had a sequence $\varepsilon_{n} \rightarrow 0$ with

$$
\delta \leqslant\left\|S_{n}-\frac{1}{\pi} J\left(u_{\varepsilon_{n}}\right)\right\|_{1,4}^{*} \leqslant \delta_{0} .
$$

This implies that $\frac{1}{\pi} J\left(u_{\varepsilon}\right) \rightarrow S_{n}$. As for

$$
\operatorname{supp}\left(S_{1}\right) \subset\left\{x \in \Omega: \operatorname{dist}\left(x, N_{1 / 2}^{\varepsilon}\right) \leqslant \eta\right\},
$$

this is a direct consequence of Proposition 4.6 from [13]. This concludes the proof of Theorem 1.1.

\section{Lower bound for $H_{c_{1}}$}

In this section we seek a lower bound for $H_{c_{1}}$ by directly analyzing the global minimizers for $G_{\varepsilon}$. As in the previous sections we assume

$$
h_{\text {ap }}=\lambda|\ln \varepsilon| \hat{e}_{3} .
$$

We again consider $h_{\text {ap }}^{\varepsilon}=|\ln \varepsilon| h_{\text {ap }}$, and $h_{\text {ap }}=\lambda \hat{e}_{3}=\nabla \times A_{\text {ap }}, A_{\text {ap }}=\nabla \times B_{\text {ap }}+\nabla \phi_{\text {ap }}, B_{\text {ap }}^{\varepsilon}=|\ln \varepsilon| B_{\text {ap }}, A_{\text {ap }}^{\varepsilon}=|\ln \varepsilon| A_{\text {ap }}$, as in Section 5.

Theorem 6.1. Let $\left(B^{*}, h^{*}\right)$ be the solution to London's equation given by Theorem 3.1. Assume that $\lambda\left\|B^{*}\right\|_{\infty}<\frac{1}{2}$. Then for a family of global minimizers of $\mathcal{G}_{\varepsilon}$, denoted by $\left(u_{\varepsilon}, A_{\varepsilon}\right)$, we have

$$
\lim _{\varepsilon \rightarrow 0} \frac{E_{\varepsilon}\left(u_{\varepsilon}\right)}{\ln (1 / \varepsilon)}=0 .
$$

In particular, the associated Jacobians $J u_{\varepsilon} \rightarrow 0$ in the strong topology on $\left(C_{T}^{0, \alpha}(\Omega)\right)^{*}$, for all $\alpha \in(0,1]$.

Remark 6.2. Note that the last statement, $J u_{\varepsilon} \rightarrow 0$, follows from (6.2) and the estimate of Proposition 5.1. In this sense we say that for applied fields $h_{\text {ap }}$ of the form (6.1) with $\lambda\left\|B^{*}\right\|_{\infty}<\frac{1}{2}$, minimizers for small $\varepsilon$ have no vortices. It has been proven that minimizers $u_{\varepsilon}$ of the energy $E_{\varepsilon}$ with prescribed Dirichlet condition have $\left|u_{\varepsilon}\right| \geqslant \frac{1}{2}$ in any neighborhood away from support of the limiting Jacobian (see [17,4].) For our problem this "Clearing Out" lemma remains an open question, although there has been recent progress on some related problems by Chiron [6].

Remark 6.3. From the above remarks we may therefore interpret Theorem 6.1 as giving a bound from below for the lower critical field $H_{c_{1}}$ in the form (6.1) with

$$
\lambda=\lambda_{m}^{*}:=\frac{1}{2\left\|B^{*}\right\|_{\infty}}=\frac{\sinh (R)}{3(\psi(R)-\psi(0))},
$$

where

$$
\psi(r)=\frac{1}{r^{2}}\left(\frac{1+r^{2}}{r} \sinh r-\cosh r\right) .
$$

We compare this with the estimate for $H_{c_{1}}$ from Section 4, which is given by

$$
\lambda^{*}=\frac{\sinh R}{3 \int_{0}^{R}(\cosh r-(\sinh r) / r) \mathrm{d} r} .
$$

A direct computation shows that

$$
\lambda_{m}^{*}=\frac{\sinh R}{3 \sum_{k \geqslant 1}((2 k+2) /(2 k+3)) R^{2 k+1} /(2 k+1) !} \leqslant \frac{\sinh R}{3 \sum_{k \geqslant 1}(2 k /(2 k+1)) R^{2 k+1} /(2 k+1) !}=\lambda^{*},
$$

and from here it is not hard to conclude that

$$
\lim _{R \rightarrow \infty} \lambda^{*}=\lim _{R \rightarrow \infty} \lambda_{m}^{*}=\frac{1}{3}
$$


and

$$
\lambda^{*}-\lambda_{m}^{*}=\mathrm{O}\left(\frac{1}{R}\right)
$$

as $R \rightarrow \infty$.

Proof. Let $\left(u_{\varepsilon}, A_{\varepsilon}\right)$ be a family of absolute minimizers of $\mathcal{G}_{\varepsilon}$, with $u_{\varepsilon} \in W^{1,2}(\Omega ; \mathbb{C})$ and $A_{\varepsilon}=A_{\text {ap }}^{\varepsilon}+A_{1, \varepsilon}$ with $A_{1, \varepsilon} \in H_{0}$. We use Lemma 2.4 as usual to write

$$
\begin{aligned}
& A_{1, \varepsilon}=\nabla \times B_{1, \varepsilon}+\nabla \phi_{\varepsilon} \quad \text { in } \Omega, \\
& B_{\varepsilon} \times v=0 \quad \text { on } \partial \Omega .
\end{aligned}
$$

Step 1. A first upper estimate for $E_{\varepsilon}\left(u_{\varepsilon}\right)$ is given by

$$
E_{\varepsilon}\left(u_{\varepsilon}\right) \leqslant \int_{\Omega} j\left(u_{\varepsilon}\right) \cdot \nabla \times\left(B_{1, \varepsilon}+B_{\mathrm{ap}}^{\varepsilon}\right) \mathrm{d} x+\int_{\Omega}\left(1-\left|u_{\varepsilon}\right|^{2}\right)\left|\nabla \times\left(B_{1, \varepsilon}+B_{\mathrm{ap}}^{\varepsilon}\right)\right|^{2} .
$$

This is an easy consequence of the fact that $\left(u_{\varepsilon}, A_{\varepsilon}\right)$ are global minimizers and hence

$$
\mathcal{G}_{\varepsilon}\left(u_{\varepsilon}, A_{1, \varepsilon}+A_{\text {ap }}^{\varepsilon}\right) \leqslant \mathcal{G}_{\varepsilon}\left(1, A_{1, \varepsilon}+A_{\text {ap }}^{\varepsilon}\right),
$$

where 1 represents the trivial constant function on $\Omega$.

Step 2. Let $h_{0}=\lambda h^{*}, A_{0}=\lambda A^{*}, B_{0}=\lambda B^{*}$, with $h^{*}, B^{*}$ as in (3.4)-(3.6), the minimizers of the magnetic energy. Write $A_{\varepsilon}=A_{1, \varepsilon}+A_{\text {ap }}^{\varepsilon}=|\ln \varepsilon| A_{0}+A_{m}, A_{m}=\nabla \times B_{m}+\nabla \phi_{m}$ as usual, and

$$
B_{\varepsilon}=B_{1, \varepsilon}+B_{\text {ap }}^{\varepsilon}=|\ln \varepsilon| B_{0}+B_{m}=\lambda_{\text {ap }}|\ln \varepsilon| B^{*}+B_{m} .
$$

From (5.2) we have

$$
\begin{aligned}
\mathcal{G}_{\varepsilon}\left(u_{\varepsilon}, A_{\varepsilon}\right)= & E_{\varepsilon}\left(u_{\varepsilon}\right)-2 \int_{\Omega} B_{\varepsilon} \cdot J\left(u_{\varepsilon}\right)+\frac{1}{2} \int_{\Omega}\left|\nabla \times\left(|\ln \varepsilon| B_{0}+B_{m}\right)\right|^{2} \\
& +\frac{1}{2} \int_{\Omega}\left(|u|^{2}-1\right)\left|\nabla \times B_{\varepsilon}\right|^{2}+\frac{1}{2} \int_{\mathbb{R}^{3}}\left|\nabla \times\left[|\ln \varepsilon|\left(A_{0}-\lambda \hat{e}_{3}\right)+A_{m}\right]\right|^{2} \\
= & E_{\varepsilon}\left(u_{\varepsilon}\right)-|\ln \varepsilon| \int_{\Omega} j\left(u_{\varepsilon}\right) \cdot \nabla \times\left(B_{0}+\frac{1}{|\ln \varepsilon|} B_{m}\right) \mathrm{d} x-\frac{1}{2} \int_{\Omega}\left(1-\left|u_{\varepsilon}\right|^{2}\right)\left|\nabla \times B_{\varepsilon}\right|^{2} \mathrm{~d} x \\
& +\frac{|\ln \varepsilon|^{2}}{2}\left\{\int_{\Omega}\left|\nabla \times B_{0}\right|^{2} \mathrm{~d} x+\int_{\mathbb{R}^{3}}\left|\nabla \times A_{0}-\lambda \hat{e}_{3}\right|^{2} \mathrm{~d} x\right\} \\
& +\frac{1}{2} \int_{\Omega}\left|\nabla \times B_{m}\right|^{2} \mathrm{~d} x+\frac{1}{2} \int_{\mathbb{R}^{3}}\left|\nabla \times A_{m}\right|^{2} \mathrm{~d} x,
\end{aligned}
$$

since two cross-terms in the expansion of the squares cancel using the critical point condition satisfied by $B_{0}$,

$$
\int_{\Omega} \nabla \times B_{0} \cdot \nabla \times B_{m} \mathrm{~d} x+\int_{\mathbb{R}^{3}}\left(\nabla \times A_{0}-\lambda \hat{e}_{3}\right) \cdot \nabla \times A_{m} \mathrm{~d} x=0 .
$$

Step 3. At this point we require the following extension of Theorem 2 from [22]:

Theorem 6.4. Let $u_{\varepsilon} \in W^{1,2}(\Omega ; \mathbb{C})$ satisfy $E_{\varepsilon}\left(u_{\varepsilon}\right) \leqslant N_{\varepsilon}|\ln \varepsilon|$, where $\delta \leqslant N_{\varepsilon} \leqslant|\ln \varepsilon|$ for some $\delta>0$ and $\left\|u_{\varepsilon}\right\|_{\infty} \leqslant C$. Then up to a subsequence: 


$$
\begin{aligned}
& \left.\left.\frac{1}{N_{\varepsilon}} J\left(u_{\varepsilon}\right) \rightarrow \bar{J} \quad \text { in the norm of }\left(C_{T}^{0, \alpha}\left(\Omega ; \mathbb{R}^{3}\right)\right)^{*} \text { for any } \alpha \in\right] 0,1\right] \\
& \frac{1}{\sqrt{N_{\varepsilon}|\ln \varepsilon|}} j\left(u_{\varepsilon}\right) \rightarrow \bar{j} \\
& \liminf _{\varepsilon \rightarrow 0} \frac{E_{\varepsilon}\left(u_{\varepsilon}\right)}{N_{\varepsilon}|\ln \varepsilon|} \geqslant M(\bar{J})+\frac{1}{2} \int_{\Omega}|\bar{j}|^{2} \mathrm{~d} x
\end{aligned}
$$

The conclusions of Theorem 6.4 are identical to those of [22], except there the result is proven for currents acting on compactly supported vector fields, $B \in C_{c}^{0, \alpha}\left(\Omega ; \mathbb{R}^{3}\right)$. The modifications required are non-trivial, and we include a proof of Theorem 6.4 in Section 7.

Using the trivial estimate, $\mathcal{G}_{\varepsilon}\left(u_{\varepsilon}, A_{\varepsilon}\right) \leqslant \mathcal{G}_{\varepsilon}\left(1, A_{\text {ap }}\right) \leqslant C|\ln \varepsilon|^{2}$ and the definition $\mathcal{G}_{\varepsilon}\left(u_{\varepsilon}, A_{\varepsilon}\right)=G_{\varepsilon}\left(u_{\varepsilon} \mathrm{e}^{\mathrm{i} \phi_{A_{\varepsilon}}}, A_{\varepsilon}\right)$, we conclude that $\int_{\mathbb{R}^{3}}\left|\nabla \times A_{\varepsilon}-h_{\mathrm{ap}}\right|^{2}=\mathrm{O}\left(|\ln \varepsilon|^{2}\right)$. Decomposing the vector potential as usual, $A_{\varepsilon}=A_{1, \varepsilon}+A_{\mathrm{ap}}^{\varepsilon}$, we then have $\left\|A_{1, \varepsilon}\right\|_{H_{0}}=\mathrm{O}(|\ln \varepsilon|)$. By Theorem 2.4, there exist $B_{\varepsilon}, \phi_{\varepsilon}$ with $\left\|\phi_{\varepsilon}\right\|_{W^{1,2}(\Omega)} \leqslant C|\ln \varepsilon|$. The cross-term in (5.2) is then estimated by Cauchy-Schwartz,

$$
\begin{aligned}
\left|\int_{\Omega} B_{\varepsilon} \cdot J\left(u_{\varepsilon}\right)\right| & =\left|\int_{\Omega}\left(A_{\varepsilon}-\nabla \phi_{\varepsilon}\right) \cdot j\left(u_{\varepsilon}\right)\right| \leqslant\left(\left\|A_{\varepsilon}\right\|_{L^{2}(\Omega)}+\left\|\phi_{\varepsilon}\right\|_{W^{1,2}(\Omega)}\right)^{2}+\frac{1}{4} \int_{\Omega}\left|\nabla u_{\varepsilon}\right|^{2} \\
& \leqslant \frac{1}{2} E_{\varepsilon}\left(u_{\varepsilon}\right)+\mathrm{O}\left(|\ln \varepsilon|^{2}\right) .
\end{aligned}
$$

The desired bound $E_{\varepsilon}\left(u_{\varepsilon}\right) \leqslant C|\ln \varepsilon|^{2}$ then follows from the definition (5.2) and the above estimates.

Recall now that critical points of the Ginzburg Landau energy satisfy $\left|u_{\varepsilon}\right| \leqslant 1$ in $\Omega$. Applying Theorem 6.4 with $N_{\varepsilon}=|\ln \varepsilon|$, we obtain a subsequence (which we continue to denote by $\varepsilon$ ) such that

$$
\frac{1}{|\ln \varepsilon|} J\left(u_{\varepsilon}\right) \rightarrow \bar{J} \quad \text { in }\left(C_{T}^{0, \alpha}\right)^{*}, \quad \frac{1}{|\ln \varepsilon|} j\left(u_{\varepsilon}\right) \rightarrow \bar{j} \quad \text { in } L^{2}\left(\Omega ; \mathbb{R}^{3}\right) .
$$

In addition, (6.6) implies:

$$
\liminf _{\varepsilon \rightarrow 0} \frac{E_{\varepsilon}\left(u_{\varepsilon}\right)}{|\ln \varepsilon|^{2}} \geqslant M(\bar{J})+\frac{1}{2} \int_{\Omega}|\bar{j}|^{2} \mathrm{~d} x .
$$

Step 4. Note that $\mathcal{G}_{\varepsilon}\left(u_{\varepsilon}, A_{\varepsilon}\right) \leqslant \mathcal{G}_{\varepsilon}\left(1,|\ln \varepsilon| A_{0}\right)$. Applying the decomposition (6.3),

$$
\begin{aligned}
& E_{\varepsilon}\left(u_{\varepsilon}\right)-\int_{\Omega} j\left(u_{\varepsilon}\right) \cdot \nabla \times B_{m} \mathrm{~d} x+\frac{1}{2} \int_{\Omega}\left|\nabla \times B_{m}\right|^{2} \mathrm{~d} x+\frac{1}{2} \int_{\mathbb{R}^{3}}\left|\nabla \times A_{m}\right|^{2} \mathrm{~d} x \\
& \leqslant|\ln \varepsilon| \int_{\Omega} j\left(u_{\varepsilon}\right) \cdot \nabla \times B_{0}+\frac{1}{2} \int_{\Omega}\left(1-\left|u_{\varepsilon}\right|^{2}\right)\left|\nabla \times B_{\varepsilon}\right|^{2} \mathrm{~d} x \\
& \quad \leqslant|\ln \varepsilon| \int_{\Omega} j\left(u_{\varepsilon}\right) \cdot \nabla \times B_{0}+C \varepsilon|\ln \varepsilon|^{3},
\end{aligned}
$$

by (5.13). Dividing the above inequality by $|\ln \varepsilon|^{2}$, and using the boundedness of $j\left(u_{\varepsilon}\right) /|\ln \varepsilon|$ we conclude that (along a subsequence)

$$
\begin{aligned}
& \frac{1}{|\ln \varepsilon|} A_{m} \rightarrow A_{m}^{*} \quad \text { in } H_{0}, \\
& \frac{1}{|\ln \varepsilon|} B_{m} \rightarrow B_{m}^{*} \quad \text { in } W_{T}^{2,2},
\end{aligned}
$$

and $\operatorname{div}\left(B_{m}^{*}\right)=0$. Moreover, by the Sobolev embedding,

$$
\frac{1}{|\ln \varepsilon|} B_{m} \rightarrow B_{m}^{*}, \quad \text { strong in } C^{0, \beta} \text { for } \beta=\frac{1}{4} \text {. }
$$


Dividing again (6.7) by $|\ln \varepsilon|^{2}$ and letting $\varepsilon \rightarrow 0$ one obtains

$$
M(\bar{J})-2 \bar{J}\left(B_{0}\right)+\frac{1}{2} \int_{\Omega}\left|\bar{j}-\nabla \times B_{m}^{*}\right|^{2} \mathrm{~d} x+\frac{1}{2} \int_{\mathbb{R}^{3}}\left|\nabla \times A_{m}^{*}\right|^{2} \mathrm{~d} x \leqslant 0 .
$$

Now we use our hypothesis

$$
\left\|B_{0}\right\|_{\infty}=\lambda\left\|B^{*}\right\|_{\infty}<\frac{1}{2}
$$

which in particular implies that $2 \bar{J}\left(B_{0}\right)<M(\bar{J})$ for $\bar{J} \neq 0$, and then from the above we obtain

$$
M(\bar{J})=\int_{\Omega}|\bar{j}|^{2} \mathrm{~d} x=\int_{\mathbb{R}^{3}}\left|\nabla \times A_{m}^{*}\right|^{2} \mathrm{~d} x=0 .
$$

In particular $A_{m}^{*}=0$ implies in addition that $B_{m}^{*}=0$.

Step 5. Set

$$
N_{\varepsilon}=\frac{1}{|\ln \varepsilon|} E_{\varepsilon}\left(u_{\varepsilon}\right)
$$

We claim that $N_{\varepsilon} \rightarrow 0$ as $\varepsilon \rightarrow 0$. To see this assume that $N_{\varepsilon_{n}} \geqslant \alpha>0$ for some $\varepsilon_{n} \rightarrow 0$.

Applying (6.8) and (6.4) we have

$$
\lim _{\varepsilon \rightarrow 0} \int_{\Omega} \frac{J\left(u_{\varepsilon}\right)}{N_{\varepsilon}} \cdot \frac{B_{m}}{|\ln \varepsilon|} \mathrm{d} x=\int_{\Omega} \bar{J} \cdot B_{m}^{*} \mathrm{~d} x=0,
$$

since $\lambda<\lambda_{m}^{*}$ implies $B_{m}^{*}=0$ by Step 4 . Dividing (6.7) by $N_{\varepsilon}|\ln \varepsilon|$, we then have:

$$
1=\frac{E_{\varepsilon}\left(u_{\varepsilon}\right)}{N_{\varepsilon}|\ln \varepsilon|} \leqslant 2 \int_{\Omega} \frac{J\left(u_{\varepsilon}\right)}{N_{\varepsilon}} \cdot \frac{B_{m}}{|\ln \varepsilon|} \mathrm{d} x+2 \int_{\Omega} \frac{J\left(u_{\varepsilon}\right)}{N_{\varepsilon}} \cdot B_{0}+\frac{C \varepsilon|\ln \varepsilon|^{2}}{N_{\varepsilon}} \rightarrow \bar{J}\left(B_{0}\right)=0,
$$

a contradiction. Therefore we must have $N_{\varepsilon} \rightarrow 0$ and the theorem is proven.

\section{Proofs of some technical results}

We include here a direct proof, for $\Omega=B_{R}(0)$, of the

Lemma 7.1. There are constants $C_{1}, C_{2}=C_{1}(\Omega), C_{2}(\Omega)$ such that for any $A \in L^{2}\left(\Omega ; \mathbb{R}^{3}\right)$ one can find a pair $\left(\phi_{A}, B_{A}\right) \in W^{1,2}(\Omega) \times W_{T}^{1,2}\left(\Omega ; \mathbb{R}^{3}\right)$ satisfying

$$
\begin{aligned}
& A=\nabla \times B_{A}+\nabla \phi_{A} \quad \text { in } \Omega \quad \text { and } \\
& \left\|\phi_{A}\right\|_{W^{1,2}}+\left\|B_{A}\right\|_{W_{T}^{1,2}} \leqslant C_{1}\|A\|_{L^{2}(\Omega)} .
\end{aligned}
$$

The choice of $B_{A}$ is unique among divergence free vector fields and the choice of $\phi_{A}$ is unique among functions in $W^{1,2}(\Omega)$ with zero average. Moreover, when $A \in H$ one also has

$$
\left\|B_{A}\right\|_{W^{2,2}}^{2}+\left\|\phi_{A}\right\|_{W^{2,2}}^{2} \leqslant C_{2}\|A\|_{H} .
$$

Proof. We first minimize the functional

$$
F(B)=\int_{\Omega}|\nabla \times B-A|^{2} \mathrm{~d} x+\int_{\Omega}(\operatorname{div} B)^{2} \mathrm{~d} x
$$

for $B \in W_{T}^{1,2}\left(\Omega ; \mathbb{R}^{3}\right)$. By Remark 2.3, a minimizing sequence for this functional in $W_{T}^{1,2}\left(\Omega ; \mathbb{R}^{3}\right)$ will be bounded in the norm of this space. We can always then extract a convergent subsequence. The lower semi-continuity of the norm in this space, and the strict convexity of the functional, guarantee that a minimizer exists and that it is unique in $W_{T}^{1,2}\left(\Omega ; \mathbb{R}^{3}\right)$. 
The critical point condition in this case reads

$$
\int_{\Omega}\left\{\left(\nabla \times B_{A}-A\right) \cdot \nabla \times B+\operatorname{div} B_{A} \cdot \operatorname{div} B\right\} \mathrm{d} x=0
$$

for all $B \in W_{T}^{1,2}\left(\Omega ; \mathbb{R}^{3}\right)$. We claim that $\operatorname{div} B_{A}=0$. To see this solve Poisson's equation

$$
\begin{aligned}
& -\Delta \phi=\operatorname{div} B_{A} \quad \text { in } \Omega, \\
& \phi=0 \quad \text { on } \partial \Omega .
\end{aligned}
$$

Since $B_{A} \in W_{T}^{1,2}\left(\Omega ; \mathbb{R}^{3}\right)$, then $\phi \in W^{2,2}(\Omega)$ so $\nabla \phi \in W^{1,2}\left(\Omega ; \mathbb{R}^{3}\right)$. On the other hand $\phi$ is constant on $\partial \Omega$. In particular $\nabla \phi \times v=0$ on $\partial \Omega$. This allows us to set $B=\nabla \phi$ in (7.4) to easily conclude that div $B_{A}=0$ a.e in $\Omega$. This reduces the critical point condition to

$$
\int_{\Omega}\left\{\nabla \times B_{A}-A\right\} \cdot \nabla \times B=\int_{\Omega} B \cdot \nabla \times\left(\nabla \times B_{A}-A\right) \mathrm{d} x=0
$$

for every $B \in W_{T}^{1,2}\left(\Omega ; \mathbb{R}^{3}\right)$. This implies that as distributions $\nabla \times\left(\nabla \times B_{A}-A\right)=0$, and since $\Omega$ is simply-connected there is a function $\phi_{A}$ satisfying $A=\nabla \times B_{A}+\nabla \phi_{A}$.

Note now that $F\left(B_{A}\right) \leqslant F(0)$ so that

$$
\int_{\Omega}\left|\nabla \times B_{A}-A\right|^{2} \mathrm{~d} x=\int_{\Omega}\left(\left|\nabla \times B_{A}\right|^{2}-2 A \cdot \nabla \times B_{A}+|A|^{2}\right) \mathrm{d} x \leqslant \int_{\Omega}|A|^{2} \mathrm{~d} x .
$$

This implies through Hölder's inequality that $\left\|\nabla \times B_{A}\right\|_{L^{2}} \leqslant C\|A\|_{L^{2}}$. Since $\operatorname{div}\left(B_{A}\right)=0$, we obtain from here

$$
\left\|B_{A}\right\|_{W_{T}^{1,2}} \leqslant C\|A\|_{L^{2}} \text {. }
$$

Now add a constant to $\phi_{A}$ so that $\int_{\Omega} \phi_{A} \mathrm{~d} x=0$. Since $\nabla \phi_{A}=A-\nabla \times B_{A}$, this and Poincaré inequality applied to $\phi_{A}$, give

$$
\left\|\phi_{A}\right\|_{W^{1,2}} \leqslant C\left\|A-\nabla \times B_{A}\right\|_{L^{2}} \leqslant C\|A\|_{L^{2}},
$$

where the last inequality is due to (7.5). It follows that

$$
\left\|B_{A}\right\|_{W_{T}^{1,2}}+\left\|\phi_{A}\right\|_{W^{1,2}} \leqslant C_{1}\|A\|_{L^{2}} \text {. }
$$

The uniqueness of $B_{A}$ can be seen as follows. If there were

$$
A=\nabla \times B_{1}+\nabla \phi_{1}=\nabla \times B_{2}+\nabla \phi_{2}
$$

with $\operatorname{div} B_{j}=0$ and $B_{j} \times v=0$ on $\partial B_{R}(0)$, then $\psi=\phi_{1}-\phi_{2}$ will satisfy

$$
\Delta \psi=\operatorname{div}\left(\nabla \phi_{1}-\nabla \phi_{2}\right)=\operatorname{div}\left(\nabla \times B_{2}-\nabla \times B_{1}\right)=0 .
$$

Moreover, a direct computation shows that, in spherical coordinates, one has

$$
(\nabla \times B) \cdot v=\frac{1}{r \sin \theta} \frac{\partial}{\partial \theta}\left(\sin \theta\left(B \cdot \hat{e}_{\theta}\right)\right)-\frac{1}{r \sin \theta} \frac{\partial}{\partial \phi}\left(B \cdot \hat{e}_{\phi}\right) .
$$

Again, direct computation reveals that $B \times v=0$ on $\partial B_{R}(0)$ implies $B \cdot \hat{e}_{\theta}=B \cdot \hat{e}_{\phi}=0$ on $\partial B_{R}(0)$. We conclude through (7.6) that

$$
B \times v=0 \text { on } \partial B_{R}(0) \Rightarrow v \cdot \nabla \times B=0 \text { on } \partial B_{R}(0) .
$$

Since $\nabla \psi=\nabla \times B_{2}-\nabla \times B_{1}$ and $B_{j} \times v=0$ on $\partial B_{R}(0)$ for $j=1,2$, we conclude that $\nabla \psi \cdot v=0$ on $\partial B_{R}(0)$. This and $\Delta \psi=0$ imply $\psi=$ const., so $\nabla \times\left(B_{1}-B_{2}\right)=0$.

We appeal now to the fact that $B_{R}(0)$ is simply-connected to find a function $\psi_{0}$ with $B_{1}-B_{2}=\nabla \psi_{0}$. This imposes $\nabla \psi_{0} \times v=0$. Hence $\nabla \psi_{0}$ is normal to $\partial B_{R}(0)$, and so $\partial B_{R}(0)$ is in fact a level set of $\psi_{0}$. Also recall that $\operatorname{div}\left(B_{j}\right)=0$ for $j=1,2$. Then $\Delta \psi_{0}=\operatorname{div}\left(B_{1}-B_{2}\right)=0$ and this implies that $\psi_{0}$ is constant in $B_{R}(0)$ as well. It follows that $B_{1}=B_{2}$. 
That $\phi_{A}$ is determined up to a constant follows because $\nabla \phi_{A}=A-\nabla \times B_{A}$, and the right-hand side of this equation is completely determined as a function of $A$.

In case $A \in H$, the estimate of $\left\|B_{A}\right\|_{W^{2,2}}$ in terms of $\|\nabla \times A\|_{L^{2}}$ comes from standard elliptic theory. To see this note first that

$$
\nabla \times \nabla \times B_{A}=-\Delta B_{A}
$$

since $\operatorname{div}\left(B_{a}\right)=0$, so the interior estimate follows. Moreover, the usual spherical coordinates for $\mathbb{R}^{3}$ make the boundary of $B_{R}(0)$ look flat. We use this fact to find equations for $B_{r}=B_{A} \cdot \hat{e}_{r}, B_{\theta}=B_{A} \cdot \hat{e}_{\theta}$ and $B_{\phi}=B_{A} \cdot \hat{e}_{\phi}$ near the boundary, along with boundary conditions for these three quantities on $\partial B_{R}(0)$. Indeed, a tedious but straightforward computation shows that

$$
-\hat{e}_{r} \cdot \nabla \times \nabla \times B_{A}=\Delta B_{r}+\frac{2}{r^{2}} \frac{\partial}{\partial r}\left(r B_{r}\right),
$$

so $B_{r}$ satisfies

$$
-\Delta B_{r}-\frac{2}{r^{2}} \frac{\partial}{\partial r}\left(r B_{r}\right)=\hat{e}_{r} \cdot \nabla \times A .
$$

Note that the right-hand side of this equation is in $L^{2}(\Omega)$. Moreover, since $B_{\theta}=B_{\phi}=0$ on the boundary of $B_{R}(0)$, the condition $\operatorname{div}\left(B_{A}\right)=0$ reduces on $\partial \Omega$ to

$$
\nabla \cdot B_{A}=\frac{1}{r^{2}} \frac{\partial}{\partial r}\left(r^{2} B_{r}\right)=\frac{\partial}{\partial r}\left(B_{r}\right)+\frac{2}{r} B_{r}=0 .
$$

This mixed boundary value problem for $B_{r}$ gives

$$
\left\|B_{r}\right\|_{W^{2,2}} \leqslant C_{2}\|\nabla \times A\|_{L^{2}} \leqslant C\|A\|_{H} .
$$

For $B_{\theta}$ and $B_{\phi}$ one obtains similar equations, but homogeneous Dirichlet boundary conditions instead.

To estimate finally $\left\|\phi_{A}\right\|_{W^{2,2}}$ we note first that (2.2) along with $A \in H$ imply $A \in W^{1,2}\left(\Omega ; \mathbb{R}^{3}\right)$. Since $\nabla \phi_{A}=$ $A-\nabla \times B_{A}$ and $B_{A} \in W^{2,2}\left(\Omega ; \mathbb{R}^{3}\right)$, we conclude that $\nabla \phi_{A} \in W^{1 / 2,2}\left(\partial \Omega ; \mathbb{R}^{3}\right)$. This plus the fact $\Delta \phi_{A}=\operatorname{div}(A)$ in $\Omega$ together allow us to conclude $\left\|\phi_{A}\right\|_{W^{2,2}} \leqslant C_{2}\|A\|_{W^{1,2}} \leqslant C\|A\|_{H}$.

Finally we give here a proof of Theorem 6.4. We need to consider vector fields that do not necessarily vanish on the boundary of $\Omega$, but rather satisfy $B \times v=0$ on $\partial \Omega$.

Proof. The only facts that need proof are that the convergence in (6.4) is in the norm of $\left(C_{T}^{0, \alpha}\left(\Omega ; \mathbb{R}^{3}\right)\right)^{*}$ rather than $\left(C_{0}^{0, \alpha}\left(\Omega ; \mathbb{R}^{3}\right)\right)^{*}$, and that the inequality in (6.6) holds here if we consider

$$
M_{T}(\bar{J})=\sup _{\left\{B \in C_{T}^{\infty}\left(\Omega ; \mathbb{R}^{3}\right):\|B\|_{\infty} \leqslant 1\right\}} \bar{J}(B) .
$$

In order to prove these statements we first introduce some notation and recall some known results. First let us recall from Lemma 7 of [12] that for any $\lambda>1$ there are constants $C_{\varepsilon}, \alpha>0$ such that for any open set $U \subset \mathbb{R}^{2}$ and $u \in H^{1}(U ; \mathbb{C})$,

$$
\left|\int_{U} \phi J u \mathrm{~d} x\right| \leqslant \lambda \int_{U}|\phi| \frac{e_{\varepsilon}(u)}{|\ln \varepsilon|} \mathrm{d} x+C_{\varepsilon} \varepsilon^{\alpha}\left(\left(1+\|\nabla \phi\|_{\infty}\right)(1+\operatorname{Meas}(\operatorname{supp}(\phi)))\right)
$$

for all functions $\phi \in C_{c}^{0,1}(U)$. Let us recall from [14] and [13] that for $\Omega \subset \mathbb{R}^{3}$ there is $C(\Omega)>0$ such that, for any $B \in C_{T}^{0,1}\left(\Omega ; \mathbb{R}^{3}\right)$,

$$
\left|\int_{\Omega} B \wedge J(u)\right| \leqslant C(\Omega) \frac{E_{\varepsilon}(u)}{|\ln \varepsilon|}\|B\|_{\infty}+C_{\varepsilon} \varepsilon^{\alpha}\|\nabla B\|_{\infty} .
$$

In both these last inequalities

$$
C_{\varepsilon}=\varepsilon^{\gamma}+\frac{E_{\varepsilon}(u)}{|\ln \varepsilon|}
$$


for some $\gamma>0$. How big $\alpha>0$ and $\gamma>0$ for us will be irrelevant, so long as they are fixed and strictly positive. Finally let us recall from [13] that for any $\Omega \subset \mathbb{R}^{3}$ and $\left.\left.\alpha \in\right] 0,1\right]$ there are constant $C(\Omega, \alpha)>0$ and $\gamma>0$ with

$$
\|J(u)\|_{C_{T}^{0, \alpha}\left(\Omega ; \mathbb{R}^{3}\right)^{*}} \leqslant C(\Omega, \alpha)\left(\varepsilon^{\gamma}+\frac{E_{\varepsilon}(u)}{|\ln \varepsilon|}\right) .
$$

We will first prove that the inequality in Theorem 6.4 is still valid if we use the mass $M_{T}(T)$ defined by (7.8). To this end assume we have a family $\left\{u_{\varepsilon}\right\}_{\varepsilon \in] 0,1]} \subset W^{1,2}(\Omega ; \mathbb{C})$ with

$$
E_{\varepsilon}\left(u_{\varepsilon}\right) \leqslant N_{\varepsilon}|\ln \varepsilon|
$$

where $\delta \leqslant N_{\varepsilon} \leqslant C|\ln \varepsilon|$. It follows from (7.11) that there is a subsequence $\varepsilon_{n} \rightarrow 0$ for which $J\left(u_{\varepsilon_{n}}\right) / N_{\varepsilon_{n}}$ is convergent in the weak $*$ topology of $\left(C_{T}^{0, \alpha}\left(\Omega ; \mathbb{R}^{3}\right)^{*}\right.$. Let us call this limit $J_{0}$. Take now any $B \in C_{T}^{\infty}\left(\Omega ; \mathbb{R}^{3}\right)$. From (7.10) we obtain that

$$
J_{0}(B) \leqslant C\|B\|_{\infty} .
$$

In particular, we can find a radon measure $\mu_{1}$ with $\mu_{1}(\Omega)<\infty$, and a $\mu_{1}$-measurable function $\tau: \Omega \rightarrow \mathbb{R}^{3}$ satisfying $|\tau|=1 \mu_{1}$-almost everywhere with

$$
J_{0}(B)=\int_{\Omega} B \cdot \tau \mathrm{d} \mu_{1}
$$

for all $B \in C_{0}^{\infty}\left(\Omega ; \mathbb{R}^{3}\right)$. We can assume here that $\mu_{1}(\partial \Omega)=0$.

We take now $B \in C_{T}^{\infty}\left(\Omega ; \mathbb{R}^{3}\right)$ and note that the functional

$$
J_{\partial \Omega}(B)=J_{0}(B)-\int_{\Omega} B \cdot \tau \mathrm{d} \mu_{1}
$$

depends only on the values of $B$ on $\partial \Omega$. In fact if $B=0$ on $\partial \Omega$ we can always find $B_{n} \in C_{0}^{\infty}\left(\Omega ; \mathbb{R}^{3}\right)$ with $B_{n} \rightarrow B$ uniformly. Note that then $J_{0}\left(B_{n}\right) \rightarrow J_{0}(B)$. But then

$$
J_{\partial \Omega}(B)=J_{0}(B)-\int_{\Omega} B \cdot \tau \mathrm{d} \mu_{1}=\lim _{n \rightarrow \infty} J_{0}\left(B_{n}\right)-\int_{\Omega} B \cdot \tau \mathrm{d} \mu_{1}=\lim _{n \rightarrow \infty} \int_{\Omega}\left(B_{n}-B\right) \cdot \tau \mathrm{d} \mu_{1}=0 .
$$

Take now any function $f \in C_{0}(\partial \Omega)$, and let $B_{f}$ be any vector field with $B \times v=0$ and $B \cdot v=f$, both on $\partial \Omega$. The fact that $J_{\partial \Omega}\left(B_{f}\right)$ depends only on the values of $B_{f}$ on $\partial \Omega$ means that it really defines a (continuous) linear functional on $C_{0}(\partial \Omega)$, and hence there is a radon measure $\mu_{2}$ on $\partial \Omega$ such that

$$
J_{\partial \Omega}\left(B_{f}\right)=\int_{\partial \Omega} B_{f} \cdot v \mathrm{~d} \mu_{2} .
$$

Follows then that we can always represent $J_{0}(B)$ as

$$
J_{0}(B)=\int_{\Omega} B \cdot \tau \mathrm{d} \mu_{1}+\int_{\partial \Omega} B \cdot v \mathrm{~d} \mu_{2}
$$

for any $B \in C_{T}\left(\Omega ; \mathbb{R}^{3}\right)$. It follows from here that

$$
M_{T}\left(J_{0}\right)=\sup _{\left\{B \in C_{T}\left(\Omega ; \mathbb{R}^{3}\right):\|B\|_{\infty} \leqslant 1\right\}} J_{0}(B)=\mu_{1}(\Omega)+\mu_{2}(\partial \Omega) .
$$

On the other hand, if we consider the measures

$$
\mu_{\varepsilon}(A)=\int_{A} \frac{e_{\varepsilon}\left(u_{\varepsilon}\right)}{N_{\varepsilon}|\ln \varepsilon|} \mathrm{d} x,
$$


for $A \subset \bar{\Omega}$, the condition $E_{\varepsilon}\left(u_{\varepsilon}\right) \leqslant N_{\varepsilon}|\ln \varepsilon|$ ensures that there is a subsequence $\varepsilon_{n} \rightarrow 0$ and a radon measure $\mu$ on $\bar{\Omega}$ with $\mu_{\varepsilon} \rightarrow \mu$ as measures, that is,

$$
\lim _{\varepsilon_{n} \rightarrow 0} \int_{\Omega} f \mathrm{~d} \mu_{\varepsilon_{n}}=\int_{\bar{\Omega}} f \mathrm{~d} \mu
$$

for any continuous function $f: \bar{\Omega} \rightarrow \mathbb{R}$. We call $\mu_{I}=\mu\left\llcorner\Omega\right.$, so that $\mu_{I}(\partial \Omega)=0$, and $\mu_{B}=\mu\llcorner\partial \Omega$. Finally note that for

$$
j(u)=\frac{1}{2 \mathrm{i}}(\bar{u} \nabla u-u \nabla \bar{u})
$$

we have

$$
\left\|j\left(u_{\varepsilon}\right)\right\|_{L^{2}}^{2} \leqslant N_{\varepsilon}|\ln \varepsilon| .
$$

There is then a subsequence $\varepsilon_{n} \rightarrow 0$ and $j_{0} \in L^{2}\left(\Omega ; \mathbb{R}^{3}\right)$ with

$$
\frac{j\left(u_{\varepsilon_{n}}\right)}{\left(N_{\varepsilon_{n}}\left|\ln \varepsilon_{n}\right|\right)^{1 / 2}} \rightarrow j_{0}
$$

Theorem 2 from [22] can be easily recast as saying

$$
\mu_{1}(\Omega)+\frac{1}{2} \int_{\Omega}\left|j_{0}\right|^{2} \mathrm{~d} x \leqslant \mu_{I}(\Omega) .
$$

What we need to prove then is that

$$
\mu_{2}(\partial \Omega) \leqslant \mu_{B}(\partial \Omega),
$$

and that the convergence $J\left(u_{\varepsilon_{n}}\right) / N_{\varepsilon_{n}} \rightarrow J_{0}$ takes place in the norm of $C_{T}^{0, \alpha}\left(\Omega ; \mathbb{R}^{3}\right)$.

In order to do this we first take $\lambda>1$ and consider an open cover of $\bar{\Omega}$, which we denote by $\left\{U_{i}\right\}_{i=1}^{n+1}$, with $U_{n+1} \subset \subset \Omega$, and diffeomorphisms $\psi_{i}: U_{i} \rightarrow B_{1}(0)$ with $\psi\left(\Omega \cap U_{i}\right)=B_{1}^{+}(0)$ for $i=1, \ldots, n$. We assume that $\left\|D \psi_{i}\right\|_{\infty},\left\|J\left(\psi_{i}\right)\right\|_{\infty} \in[1 / \lambda, \lambda]$, where $J\left(\psi_{i}\right)$ denotes the classical Jacobian of $\psi_{i}$. Finally we consider $\phi_{i}: U_{i} \rightarrow$ $[0,1]$ smooth compactly supported in $U_{i}$, with

$$
\sum_{i=1}^{n+1} \phi_{i}=1
$$

on $\bar{\Omega}$.

Consider now a smooth function $f: \partial \Omega \rightarrow \mathbb{R}$ and define

$$
f_{i}(\cdot)=\phi_{i}\left(\psi_{i}^{-1}(\cdot)\right) f\left(\psi_{i}^{-1}(\cdot)\right) \text {. }
$$

Clearly $f_{i}$ is smooth and compactly supported on $\partial B_{1}^{+}(0) \cap\left\{x \in \mathbb{R}^{3}: x_{3}=0\right\}$. Let $\chi_{\delta}:[0, \delta] \rightarrow[0,1]$ with $\chi_{\delta}(0)=1$ and $\chi_{\delta}(\delta)=0$. We choose $\delta>0$ small enough so that the fields

$$
B_{i}\left(x_{1}, x_{2}, x_{3}\right)=f_{i}\left(x_{1}, x_{2}, 0\right) \chi_{\delta}\left(x_{3}\right) \hat{e}_{3}
$$

are all compactly supported in $B_{1}^{+}(0)$. Next we extend these vector fields symmetrically to all of $B_{1}(0)$ and consider $z_{i}(x)=u\left(\psi_{i}^{-1}(x)\right)$, which we also extend to all of $B_{1}(0)$ by $z_{i}\left(x_{1}, x_{2},-x_{3}\right)=z_{i}\left(x_{1}, x_{2}, x_{3}\right)$. Following [13] we note that

$$
\int_{U_{i}}\left(\psi_{i}\right)^{\#} B_{i} \cdot J(u) \mathrm{d} x \int_{B_{1}^{+}(0)} B_{i} \cdot J\left(z_{i}\right) \mathrm{d} x=\frac{1}{2} \int_{B_{1}(0)} B_{i} \cdot J\left(z_{i}\right) .
$$

For the last term in this identity we note first that

$$
\int_{B_{1}(0)} B_{i} \cdot J\left(z_{i}\right) \mathrm{d} x=\int_{-\delta}^{\delta} \chi_{\delta}(z) \int_{C_{0}} f_{i}\left(x_{1}, x_{2}\right) J_{1,2}\left(z_{i}\right)\left(x_{1}, x_{2}, z\right) \mathrm{d} x_{1} \mathrm{~d} x_{2} \mathrm{~d} z,
$$


where $J_{1,2}\left(z_{i}\right)\left(x_{1}, x_{2}, z\right)$ denotes the Jacobian of the restriction of $z_{i}$ to the plane $\left\{\left(x_{1}, x_{2}, x_{3}\right) \in \mathbb{R}^{3}: x_{3}=z\right\}$, and $C_{0}=\left\{\left(x_{1}, x_{2}, x_{3}\right) \in \mathbb{R}^{3}: x_{3}=0, x_{1}^{2}+x_{2}^{2} \leqslant 1\right\}$. We can apply then (7.10) to the inner integral in the last term to obtain

$$
\begin{aligned}
\int_{B_{1}(0)} B_{i} \cdot J\left(z_{i}\right) & \leqslant \lambda \int_{B_{1}(0)}\left|B_{i}\right| \frac{e_{\varepsilon}\left(z_{i}\right)}{|\ln \varepsilon|}+C \varepsilon^{\alpha}\left(\left(1+\left\|\nabla B_{i}\right\|_{\infty}\right)\left(1+\operatorname{Meas}\left(\operatorname{supp}\left(B_{i}\right)\right)\right)\right) \\
& =2 \lambda \int_{B_{1}^{+}(0)}\left|B_{i}\right| \frac{e_{\varepsilon}\left(z_{i}\right)}{|\ln \varepsilon|}+C \varepsilon^{\alpha}\left(1+\left\|\nabla B_{i}\right\|_{\infty}\right)\left(1+\operatorname{Meas}\left(\operatorname{supp}\left(B_{i}\right)\right)\right) .
\end{aligned}
$$

Let us now the diffeomorphisms $\psi_{i}$ to rewrite the integral over $B_{1}^{+}(0)$ in this inequality as being over $U_{i}$ instead,

$$
\int_{B_{1}^{+}(0)}\left|B_{i}\right| \frac{e_{\varepsilon}\left(z_{i}\right)}{|\ln \varepsilon|} \mathrm{d} x \leqslant \lambda^{4} \int_{U_{i}}\left|\left(\psi_{i}\right)^{\#}\left(B_{i}\right)\right| \frac{e_{\varepsilon}(u)}{|\ln \varepsilon|} .
$$

We now add $i=1, \ldots, n$ to obtain

$$
\sum_{i=1}^{n} \int_{U_{i}}\left(\psi_{i}\right)^{\#}\left(B_{i}\right) \cdot J(u) \leqslant \lambda^{4} \sum_{i=1}^{n} \int_{U_{i}}\left|\left(\psi_{i}\right)^{\#}\left(B_{i}\right)\right| \frac{e_{\varepsilon}(u)}{|\ln \varepsilon|}+C \varepsilon^{\alpha} \sum_{i=1}^{n}\left(1+\left\|\nabla B_{i}\right\|_{\infty}\right)\left(1+\operatorname{Meas}\left(\operatorname{supp}\left(B_{i}\right)\right)\right) .
$$

We now do several things: First divide by $N_{\varepsilon}$ and let $\varepsilon \rightarrow 0$. This yields

$$
J_{0}\left(\sum_{i=1}^{n}\left(\psi_{i}\right)^{\#}\left(B_{i}\right)\right) \leqslant \lambda^{4} \int_{\bar{\Omega}} \sum_{i=1}^{n}\left|\left(\psi_{i}\right)^{\#}\left(B_{i}\right)\right| \mathrm{d} \mu .
$$

Next we consider $\delta \rightarrow 0$ in the definition of $\chi_{\delta}$. Since all the measures involved are finite we can use dominated convergence to claim that both terms in this last inequality will converge to the corresponding boundary integrals. Moreover, the definition of the $B_{i}$ and the fact that

$$
\sum_{i=1}^{n} \phi_{i}=1
$$

on $\partial \Omega$ means the result of letting $\delta \rightarrow 0$ is

$$
\int_{\partial \Omega} f \mathrm{~d} \mu_{2} \leqslant \lambda^{4} \int_{\partial \Omega}|f| \mathrm{d} \mu_{B}
$$

Taking now supremum over all $|f| \leqslant 1$ on $\partial \Omega$ and letting $\lambda \rightarrow 1$ we conclude

$$
\mu_{2}(\partial \Omega) \leqslant \mu_{B}(\partial \Omega)
$$

which implies

$$
M\left(J_{0}\right)+\frac{1}{2} \int_{\Omega}\left|j_{0}\right|^{2} \mathrm{~d} x \leqslant \mu(\bar{\Omega}) .
$$

To show now that the convergence

$$
\frac{1}{N_{\varepsilon}} J\left(u_{\varepsilon}\right) \rightarrow \bar{J}
$$

is in the norm of $\left(C_{T}^{0, \alpha}\left(\Omega ; \mathbb{R}^{3}\right)\right)^{*}$ for any $\left.\left.\alpha \in\right] 0,1\right]$ we again appeal to a covering of $\bar{\Omega}$ denoted by $\left\{U_{i}\right\}_{i=1}^{n+1}$ with $U_{n+1} \Subset \Omega$ and smooth diffeomorphisms $\psi_{1}: U_{i} \rightarrow B_{1}(0)$ with $\psi_{i}\left(U_{i} \cap \Omega\right)=B_{1}^{+}(0)$. We again consider a partition of unity $\left\{\phi_{i}\right\}_{i=1}^{n+1}$ of smooth functions that satisfy

$$
\sum_{i=1}^{n+1} \phi_{i}=1
$$


on $\bar{\Omega}$. Next we compute for any $B \in C_{T}^{\infty}\left(\Omega ; \mathbb{R}^{3}\right)$,

$$
\int_{\Omega} B \cdot J(u) \mathrm{d} x=\sum_{i=1}^{n+1} \int_{U_{i}} \phi_{i} B \cdot J(u) \mathrm{d} x=\sum_{i=1}^{n} \int_{B_{1}^{+}(0)}\left(\psi_{i}^{-1}\right)^{\#}\left(\phi_{i} B\right) J\left(z_{i}\right) \mathrm{d} x+\int_{U_{n+1}} \phi_{n+1} B \cdot J(u) \mathrm{d} x .
$$

Again we follow [13] to rewrite the first $n$ integrals above as integrals over the whole unit sphere $B_{1}(0)$ as follows: Let $B_{i}=\left(\psi_{i}^{-1}\right)^{\#}\left(\phi_{i} B\right)$ and define $\tilde{x}=\left(x_{1}, x_{2}, x_{3}\right)$ and $\widetilde{B}_{i}$ by

$$
\widetilde{B}_{i}(x)= \begin{cases}B_{i}(x) & \text { for } x \in B_{+}(0,1), \\ -\left(B_{i} \cdot \hat{e}_{1}\right)(\tilde{x}) \mathrm{d} x_{1}-\left(B_{i} \cdot \hat{e}_{2}\right)(\tilde{x}) \mathrm{d} x_{2}+\left(B_{i} \cdot \hat{e}_{3}\right)(\tilde{x}) \mathrm{d} x_{3} & \text { otherwise. }\end{cases}
$$

As noted in [13] this reflection produces Lipschitz vector fields $\widetilde{B}_{i}$ in $B_{1}(0)$ because of the condition $B \times v=0$ on $\partial \Omega$. Defining $\tilde{z}_{i}(\tilde{x})=z_{i}(x)$ for $x \in B_{1}^{+}(0)$, it follows directly from these definitions that

$$
\int_{B_{1}^{+}(0)}\left(\psi_{i}^{-1}\right)^{\#}\left(\phi_{i} B\right) J\left(z_{i}\right) \mathrm{d} x=\frac{1}{2} \int_{B_{1}(0)} \widetilde{B}_{i} \cdot J\left(\tilde{z}_{i}\right),
$$

so we obtain then

$$
\int_{\Omega} B \cdot J(u) \mathrm{d} x=\sum_{i=1}^{n+1} \int_{U_{i}} \phi_{i} B \cdot J(u) \mathrm{d} x=\frac{1}{2} \sum_{i=1}^{n} \int_{B_{1}(0)} \widetilde{B}_{i} J\left(\tilde{z}_{i}\right) \mathrm{d} x+\int_{U_{n+1}} \phi_{n+1} B \cdot J(u) \mathrm{d} x .
$$

We note now that all the energy bounds for $E\left(u_{\varepsilon}\right)$ are still true for $E_{\varepsilon}\left(\tilde{z}_{i}\right)$ and hence we can apply the convergence part of Theorem 6.4 to each one of the previous terms to conclude that each of the $J\left(\tilde{z}_{i}\right)$ will converge to a $J_{0}^{i}$ in the norm of $\left(C_{0}^{0, \alpha}\left(B_{1}(0) ; \mathbb{R}^{3}\right)\right)^{*}$, and hence $J\left(u_{\varepsilon}\right) \rightarrow J_{0}$ in the norm of $\left(C_{T}^{0, \alpha}\left(\Omega ; \mathbb{R}^{3}\right)\right)^{*}$.

Remark 7.2. From the proof, we see that the effect of considering vector fields with non-vanishing boundary values is that the limiting Radon measure representing the current $J_{0}$ splits into two components, $\mu_{1}, \mu_{2}$. The component $\mu_{1}$ acts in the interior of $\Omega$, while $\mu_{2}$ acts at the boundary, along the normal direction. There are two cases where one can show that $\mu_{2}=0$. One is the case $\delta \leqslant N_{\varepsilon} \leqslant C$ as pointed out in [13]. The second is $N_{\varepsilon}=\mathrm{O}\left(|\ln \varepsilon|^{2}\right)$. To see this consider $\Omega=B_{1}^{+}(0)$ and let $f \in C_{0}^{\infty}\left(C_{0}\right)$. Here

$$
C_{0}=\left\{\left(x_{1}, x_{2}, x_{3}\right) \in \mathbb{R}^{3}: x_{1}^{2}+x_{2}^{2} \leqslant 1\right\}
$$

as before. Again, let $\chi_{\delta}:[0, \delta] \rightarrow[0,1]$ be such that $\chi_{\delta}(0)=1, \chi_{\delta}(\delta)=0$, and consider the vector field $B=\chi_{\delta} f \hat{e}_{3}$. In this case we have

$$
\begin{aligned}
\left|\int_{B_{1}^{+}(0)} B \cdot J(u) \mathrm{d} x\right| & =\left|\int_{0}^{\delta} \chi_{\delta} \int_{C_{0}} f J_{1,2}(u) \mathrm{d} x_{1} \mathrm{~d} x_{2} \mathrm{~d} z\right|=\left|\int_{0}^{\delta} \chi_{\delta} \int_{C_{0}} j_{1,2}(u) \cdot \nabla^{\perp} f \mathrm{~d} x_{1} \mathrm{~d} x_{2} \mathrm{~d} z\right| \\
& \leqslant\left\|\nabla^{\perp} f\right\|_{\infty} \int_{0}^{\delta} \chi_{\delta}\left(\int_{\operatorname{supp}(f)}\left|j_{1,2}\right|^{2}\right)^{1 / 2}\left|C_{0}\right|^{1 / 2} \mathrm{~d} z \\
& \leqslant C\left\|\nabla^{\perp} f\right\|_{\infty}\left\{\int_{0}^{\delta} \chi_{\delta} \mathrm{d} z \int_{B_{1}^{+}(0)}\left|j_{1,2}\right|^{2} \mathrm{~d} x\right\}^{1 / 2} .
\end{aligned}
$$

Here $\nabla^{\perp} f=\left(f_{x_{2}},-f_{x_{1}}\right)$ and

$$
j_{1,2}=\frac{1}{2 \mathrm{i}}\left(\bar{u} \nabla_{1,2} u-u \nabla_{1,2} \bar{u}\right)
$$


with $\nabla_{1,2} f=\left(f_{x_{1}}, f_{x_{2}}\right)$. From (7.13) we obtain, after dividing by $N_{\varepsilon}=|\ln \varepsilon|$ and letting $\varepsilon \rightarrow 0$,

$$
\left|J_{0}(B)\right| \leqslant C\left\|\nabla^{\perp} f\right\|_{\infty}\left(\int_{0}^{\delta} \chi_{\delta} \mathrm{d} z\right)^{1 / 2} .
$$

From here now letting $\delta \rightarrow 0$ we obtain

$$
\int_{\partial \Omega} f \mathrm{~d} \mu_{2}=0 .
$$

The case of a general boundary can then be treated using the flattening of the boundary argument used before.

\section{Acknowledgement}

We thank Peter Sternberg for suggesting an approach to part (ii) of Proposition 4.2.

\section{References}

[1] A. Aftalion, R. Jerrard, Properties of a single vortex solution in a rotation Bose-Einstein condensate, C. R. Acad. Sci. Paris, Ser. I 336 (2003) 713-718.

[2] G. Alberti, S. Baldo, G. Orlandi, Variational convergence for functionals of the Ginzburg-Landau type, Preprint, 2003.

[3] F. Bethuel, H. Brezis, F. Hélein, Ginzburg-Landau Vortices, Birkhäuser, Basel, 1994.

[4] F. Bethuel, H. Brezis, G. Orlandi, Asymptotics for the Ginzburg-Landau equation in arbitrary dimensions, J. Func. Anal. 186 (2001) $432-520$.

[5] F. Bethuel, T. Rivière, F. Bethuel, T. Rivière, Vortices for a variational problem related to superconductivity, Ann. Inst. H. Poincaré Anal. Non Linéaire 12 (1995) 243-303.

[6] D. Chiron, Boundary problems for the Ginzburg-Landau equation, Preprint, Commun. Cont. Math., in press.

[7] H. Federer, Geometric Measure Theory, Springer-Verlag, New York, 1969.

[8] D. Gilbarg, N. Trudinger, Elliptic Partial Differential Equations of Second Order, Revised third printing, Springer-Verlag, 1998.

[9] T. Giorgi, D. Phillips, The breakdown of superconductivity due to strong fields in the Ginzburg-Landau model, SIAM J. Math. Anal. 30 (2) (1999) 341-359.

[10] E. Giusti, Minimal Surfaces and Functions of Bounded Variation, Birkhäuser, 1984.

[11] T. Iwaniec, C. Scott, B. Stroffolini, Nonlinear Hodge theory on manifolds with boundary, Ann. Mat. Pura Appl. (4) CLXXVII (1999) 37-115.

[12] R.L. Jerrard, More about Bose Einstein condensates, Preprint, 2003.

[13] R. Jerrard, A. Montero, P. Sternberg, Local minimizers of the Ginzburg-Landau energy with magnetic field in three dimensions, Comm. Math. Phys. 249 (3) (2004) 549-577.

[14] R. Jerrard, H.M. Soner, The Jacobian and the Ginzburg-Landau energy, Calc. Var. Partial Differential Equations 14 (2) (2002) $151-191$.

[15] R.V. Kohn, P. Sternberg, Local minimizers and singular perturbations, Proc. Roy. Soc. Edinburgh Ser. A 111 (1989) 69-84.

[16] O. Ladyzhenskaya, The Mathematical Theory of Viscous Incompressible Flow, Gordon and Breach Science Publishers, 1969.

[17] F.H. Lin, T. Rivière, Complex Ginzburg-Landau equations in higher dimensions and codimension two area minimizing currents, J. Eur. Math. Soc. 1 (1999) 237-322.

[18] F. London, Superfluids, Wiley, New York, 1950-1954.

[19] A. Montero, P. Sternberg, W. Ziemer, Local minimizers with vortices to the Ginzburg-Landau system in three dimensions, CPAM LVII (2004) 0099-0125.

[20] T. Rivière, Line vortices in the $U$ (1)-Higgs model, ESAIM Control Optim. Calc. Var. 1 (1996) 77-167.

[21] E. Sandier, Ginzburg-Landau minimizers from $\mathbb{R}^{n+1}$ to $\mathbb{R}^{n}$ and minimal connections, Indiana Univ. Math. J. 50 (4) (2001) $1807-1844$.

[22] E. Sandier, S. Serfaty, A product estimate for Ginzburg-Landau and corollaries, J. Funct. Anal. 211 (1) (2004) 219-244.

[23] E. Sandier, S. Serfaty, Ginzburg-Landau minimizers near the first critical field have bounded vorticity, Calc. Var. Partial Differential Equations 17 (1) (2003) 17-28.

[24] S. Serfaty, Local minimizers for the Ginzburg-Landau energy near critical magnetic field I, Comm. Cont. Math. 1 (3) (1999) $295-333$.

[25] M. Tinkham, Superconductivity, Gordon and Breach, 1965.

[26] H. Weyl, The method of orthogonal projection in potential theory, Duke Math. J. 7 (1940) 411-444.

[27] W.P. Ziemer, Weakly Differentiable Functions, Springer-Verlag, 1989. 